Key words. Emissions markets, Cap-and-trade schemes, Equilibrium models, Environmental Finance.

\title{
MARKET DESIGN FOR EMISSION TRADING SCHEMES
}

\author{
RENÉ CARMONA *, MAX FEHR ${ }^{\dagger}$, JURI HINZ $\ddagger$, AND ARNAUD PORCHET $\S$
}

\begin{abstract}
The main thrust of the paper is the design and the numerical analysis of new capand-trade schemes for the control and the reduction of atmospheric pollution. The tools developed are intended to help policy makers and regulators understand the pros and the cons of the emissions markets. We propose a model for an economy where risk neutral firms produce goods to satisfy an inelastic demand and are endowed with permits by the regulator in order to offset their pollution at compliance time and avoid having to pay a penalty. Firms that can easily reduce emissions do so, while those for which it is harder buy permits from those firms anticipating that they will not need them, creating a financial market for pollution credits. Our model captures most of the features of the European Union Emissions Trading Scheme. We show existence of an equilibrium and uniqueness of emissions credit prices. We also characterize the equilibrium prices of goods and the optimal production and trading strategies of the firms. We choose the electricity market in Texas to illustrate numerically the qualitative properties observed during the implementation of the first phase of the European Union cap-and-trade $\mathrm{CO}_{2}$ emissions scheme, comparing the results of capand-trade schemes to the Business As Usual benchmark. In particular, we confirm the presence of windfall profits criticized by the opponents of these markets. We also demonstrate the shortcomings of tax and subsidy alternatives. Finally we introduce a relative allocation scheme which despite of its ease of implementation, leads to smaller windfall profits than the standard scheme.
\end{abstract}

1. Introduction. Emission trading schemes, also known as cap and trade systems, have been designed to reduce pollution by introducing appropriate market mechanisms. The two most prominent examples of existing cap and trade systems are the EU-ETS (European Union Emission Trading Scheme) and the US Sulfur Dioxide Trading System. In such systems, a central authority sets a limit (cap) on the total amount of pollutant that can be emitted within a pre-determined period. To ensure that this target is complied with, a certain number of credits are allocated to appropriate installations, and a penalty is applied as a charge per unit of pollutant emitted outside the limits of a given period. Firms may reduce their own pollution or purchase emission credits from a third party, in order to avoid accruing potential penalties. The transfer of allowances by trading is considered to be the core principle leading to the minimization of the costs caused by regulation: companies that can easily reduce emissions will do so, while those for which it is harder buy credits.

In a cap-and-trade system, the initial allocation (i.e. the total number of allowances issued by the regulator) should be chosen in order for the scheme to reach a given emissions level. This total initial allocation is indeed the crucial parameter that the regulator uses as a knob to control the emission level. But while the value of the total initial allocation is driven by the emissions target, the specific distribution of these allowances among the various producers and market participants can be chosen

* Department of Operations Research and Financial Engineering, Princeton University, Princeton, NJ 08544. Also with the Bendheim Center for Finance and the Applied and Computational Mathematics Program. (rcarmona@princeton.edu).

†Institute for Operations Research, ETH Zurich, CH-8092 Zurich, Switzerland (maxfehr@ifor.math.ethz.ch).

${ }^{\ddagger}$ National University of Singapore, Department of Mathematics, 2 Science Drive, 117543 Singapore (mathj@nus.edu.sg), Partially supported by WBS R-703-000-020-720 / C703000 of the Risk Management Institute at the National University of Singapore

$\S$ Timbre J320, 15 Boulevard Gabriel Pri, 92245 MALAKOFF Cedex, FRANCE, (arnaud.porchet@gmail.com). 
in order to create incentives to design and build cleaner and more efficient production units.

Naturally, emissions reduction increases the costs of goods whose production causes those emissions. Part or all of these costs are passed on to the end consumer and substantial windfall profits are likely to occur. Based on an empirical analysis of power generation profitability in the context of EU-ETS, strong empirical evidence of the existence of such profits is given in [14]. The authors of this study come to the conclusion that power companies realize substantial profits since allowances are received for free while they are always priced into electrical power at a rate that depends upon the emission rate of the marginal production unit: producers seem to take advantage of the trading scheme to make extra profit. This phenomenon can even happen in a competitive setting. What follows is a simple illustration in a deterministic framework.

Let us consider a set of firms that must satisfy a demand of $D=1 \mathrm{MWh}$ of electricity at each time $t=0,1, \cdots, T-1$, and let us assume that there are only two possible technologies to produce electricity: gas technology which has unit cost $2 \$$ and emits 1 ton of $\mathrm{CO}_{2}$ per MWh, and coal technology which has unit cost $1 \$$ and emits 2 tons of $\mathrm{CO}_{2}$ per MWh. In this simple model, the total capacity of gas is $1 \mathrm{MWh}$ and coal's capacity is also $1 \mathrm{MWh}$. We also suppose that producers face a penalty $\pi>1 \$$ per ton of $\mathrm{CO}_{2}$ not offset by credits, and that a total of $T-1$ credits are distributed to the firms, allowing them to offset altogether $T-1$ tons of $\mathrm{CO}_{2}$. In this situation, we arrive at two conclusions. First, as demand needs to be met, total emissions will be higher or equal than $T$ tons, even if all firms use the clean technology (gas). Second, firms are always better off reducing emissions than paying the penalty. As a consequence, the optimal generation strategy is to only use the gas technology and emit $T$ tons of $\mathrm{CO}_{2}$. At least one firm has to pay the penalty, and the price of emission credits is necessarily equal to $\pi$ at each time. Indeed the missing credit has a value $\pi$ for both the buyer and the seller. The price of electricity is then $2+\pi$ because a marginal decrease in demand will induce a marginal gain in generation cost and a marginal decrease of the penalty paid. The total profit for the producers is $\pi(T-1)$, the penalty paid by the producers to the regulator is $\pi$, and the total cost for the customers is $(2+\pi) T$. Consider now the Business As Usual (BAU) situation: the demand is met by using coal technology, the price of electricity is 1 , the total profit for producers is 0 and the total cost for the customers is $T$. In this simple example the producers cost induced by the trading scheme is $T+\pi$ : producers must buy more expensive fuel, so a profit $T$ is made by the fuel supplier and they have to pay the penalty $\pi$. The increase in fuel price, or switching cost, is a marginal cost that must factor into the electricity price. The penalty is a fixed cost paid at the end, but we see that in this trading scheme, this fixed cost is rolled over the entire period and paid by the customers at each time, inducing a windfall profit for the producers. This windfall profit is exactly equal to the market value of the $T-1$ credits: these credits are given for free by the regulator but their market value is actually funded by the customer.

Another feature of emissions trading schemes is the risk of non compliance faced by the producers and the regulator. The EU-ETS was introduced as a way of complying with the targets set by the Kyoto Protocol. Phase 1 of the Kyoto Protocol sets a fixed cap for annual emissions of $\mathrm{CO}_{2}$ by year 2012 to all industrialized countries that ratified the protocol (Annex I countries). This reduction should guarantee on average a level of emissions of $95 \%$ of what it was in year 1990. All countries are free to 
adopt the emission reduction policy of their choice, but in case of non-compliance in 2012, they face a penalty (payment of 1.3 emission allowances for each ton not offset in Phase 1). The EU-ETS was designed to ensure compliance for the whole EU zone. However, in an uncertain environment, there exists the possibility that the scheme will fail its goal and that the producers will exceed the fixed cap set at the beginning of the compliance period. In this case, it is the regulator's responsibility to comply with the target by buying allowances from other countries or generate additional allowances by investing in clean projects under the Clean Development Mechanism (CDM for short) or the Joint Implementation (JI for short) mechanism, or otherwise, to pay the penalty. The design of emission trading schemes must also address this question.

In the present work, we give a precise mathematical foundation to the analysis of emission trading schemes and quantitatively investigate the impact of emission regulation on consumers costs and company's profits. Based on an equilibrium model for perfect competition, we show that the action of an emission trading scheme combines two contrasting aspects. On the one hand, the system reduces pollution at the lowest cost for the society, as expected. On the other hand, it forces a notable transfer of wealth from consumers to producers, which in general exceeds the social costs of pollution reduction.

In a perfect economy where all customers are shareholders, windfall profits are redistributed, at least partially, by dividends. However, this situation is not the general case and the impact of regulation on prices should be addressed. There are several other ways to return part of the windfall profits to the consumers. The most prominent ones are taxation and charging for the initial allowance distribution. Beyond the political risks associated with new taxes, we will show that one of the main disadvantages of this first method is its poor control of emissions under stochastic abatement costs. Concerning auctioning, it is important to notice that, in the first phase of the EU-ETS, individual countries did not have to give away the totality of their credit allowances for free. They could choose to auction up to $10 \%$ of their total allowances. Strangely enough, except for Denmark, none of them exercised this option. On the other hand using auctioning as a way to abolish windfall profits, one looses one of the main features of cap-and-trade schemes, namely the mechanism which allows to control the incentives to invest in and develop cleaner production technologies. Indeed, a significant reduction of windfall profits through auctioning, if at all possible, requires that a huge amount or even the total initial allocation is auctioned. Further it involves a significant risk for companies since the capital invested to procure allowances at the auction may be higher than the income later recovered from allowances prices.

In this work, we argue that cap-and-trade schemes can work, even in the form implemented in the first phase of EU-ETS, at least as long as allowance distribution is properly calibrated. Moreover, we prove that it is possible to design modified emission trading schemes that overcome these problems. We show how to establish trading schemes that reduce windfall profits while exhibiting the same emission reduction performance as the generic cap and trade system used in the first implementation phase of the EU-ETS. These schemes also have the nice feature that a significant amount of the allowances can be allocated as initial allocation to encourage cleaner technologies.

Despite frequent articles in the popular press and numerous speculative debates in specialized magazines and talk-shows, the scientific literature on cap-and-trade 
systems is rather limited. We briefly mention a few related works chosen because of their relevance to our agenda. The authors of [3] and [9] proposed a market model for the public good environment introduced by tradable emission credits. Using a static model for a perfect market with pollution certificates, [9] shows that there exists a minimum cost equilibrium for companies facing a given environmental target. The conceptual basis for dynamic permit trading is, among others, addressed in [2], [15], [11], [7], [12] and [13]. Meanwhile, the recent work [13] suggests also a continuous-time model for carbon price formation. Beyond these themes, there exists a vast literature on several related topics, including equilibrium [1], empirical evidence from already existing markets [6], [14], and uncertainty and risk [5], [8], [16]. The model we present below follows the baseline suggested in [4].

We close this introduction with a quick summary of the contents of the paper.

Section 2 gives the details of the mathematical model used to capture the dynamic features of a cap-and-trade system. We introduce the necessary notation to describe the production of goods and the profit mechanisms in a competitive economy. Exogenous demand for goods is modeled by means of adapted stochastic processes. We assume that demand is inelastic and has to be met exactly. This assumption could be viewed as unusually restrictive, but we argue that it is quite realistic in the case of electricity. We also introduce the emissions allowance allocations and the rules of trading in these allowances.

Section 3 defines the notion of competitive equilibrium for risk neutral firms involved in our cap-and-trade scheme. Preliminary work shows that most of the theoretical results of this paper still hold for risk averse firms if preferences are modeled with exponential utility. However, in order to avoid muddying the water with unnecessary technical issues which could distract the reader from the important issues of pollution abatement, we restrict ourselves to the less technical case of risk neutral firms. For the sake of completeness, we solve the equilibrium problem in the Business As Usual (BAU from now on) case corresponding to the absence of market for emissions permits. In this case, as expected, the prices of goods are given by the standard merit order pricing typical of deregulated markets. The section closes with the proof of a couple of enlightening necessary conditions for the existence of an equilibrium in our model. These mathematical results show that at compliance time, the equilibrium price of an emission certificate can only be equal to 0 or to the penalty level chosen by the regulator. The second important necessary condition shows that in equilibrium, the prices of the goods are still given by a merit order pricing provided that the production costs are adjusted for the cost of emissions. This result is important as it shows exactly how the price of pollution gets incorporated in the prices of goods in the presence of a cap-and-trade scheme. The following Section 4 is devoted to the rigorous proof of the existence of an equilibrium. The proof uses classical functional analysis results on optimization in infinite dimensional spaces. It follows the lines of a standard argument based on the analysis of what an informed central planner (representative agent) would do in order to minimize the social cost of meeting the demand for goods.

Section 5 is devoted to the analysis of the standard cap-and-trade scheme featured in the implementation of the first phase of the EU-ETS. By comparison with BAU scenarios, we show that properly chosen levels of penalty and pollution certificate allocations lead to desired emissions targets. However, our numerical experiments on a case study of the electricity market in Texas show the existence of excessive windfall profits. As explained earlier in our literature review, these profits have been 
observed in the first phase of EU-ETS, giving credibility to the critics of cap-andtrade systems. Section 6 can be viewed as the main thrust of the paper beyond the theoretical results proven up to that point. We propose a general framework including taxes and subsidies along the standard cap-and-trade schemes. We demonstrate the shortcomings of the tax systems which suffer from poor control of the windfall profits and unexpected expensive reduction policies when it comes to emissions reduction targets under stochastic abatement costs. We concentrate our analysis on several new alternative cap-and-trade schemes and we show numerically that a relative allocation scheme can resolve most of the issues with the other schemes. Such a relative allocation scheme is easy to describe and implement as pollution allowances are distributed proportionally to production. Even though the number of permits is random in a relative scheme, and hence cannot be known in advance, its statistical distribution is well understood as it is merely a scaled version of the distribution of the demands for goods. Consequently, setting up caps to meet pollution targets is not much different from the standard cap-and-trade schemes. Moreover, the coefficient of proportionality providing the number of permits is an extra parameter which should make the calibration more efficient. Indeed, one shows that properly calibrated, the relative schemes reach the same pollution targets as the standard schemes while at the same time, they keep social costs and windfall profits in control.

Section 7 gathers more mathematical properties of the generalized cap-and-trade schemes introduced in the previous section. Our results demonstrate the versatility and the flexibility of such a generalized framework. It shows that regulators can control cap-and-trade schemes in order to reach pre-assigned pollution targets with zero windfall profits and reasonably small social costs, or even to force equilibrium electricity prices to be equal to target prices. However, because of the level of complexity of their implementations, it is unlikely that the schemes identified there will be used by policy makers or regulators. The paper concludes with Section 8 which reviews the main results of the paper recasting them in the perspective of the public policy challenging issues uncovered by the results of the paper.

2. Standard Cap-and-Trade Scheme. In this section we present the elements of our mathematical analysis. We consider an economy where a set of firms produce and supply goods to end-consumers over a period $[0, T]$. The production of these goods is a source of pollutant emissions. In order to reduce this externality, a regulator distributes emissions allowances to the firms at time 0 , allows them to trade the allowances on an organized market between times 0 and $T$, and at the end of this compliance period, taxes the firms proportionally to their net cumulative emissions.

In what follows $\left(\Omega, \mathcal{F},\left\{\mathcal{F}_{t}, t \in\{0,1, \ldots, T\}\right\}, \mathbb{P}\right)$ is a filtered probability space. We denote by $\mathbb{E}[$.$] the expectation operator under probability \mathbb{P}$ and by $\mathbb{E}_{t}[$.$] the$ expectation operator conditional to $\mathcal{F}_{t}$. The $\sigma$-field $\mathcal{F}_{t}$ represents the information available at time $t$. We will also make use of the notation $\mathbb{P}_{t}():.=\mathbb{E}_{t}\left[\mathbf{1}_{\{.\}}\right]$for the conditional probability with respect to $\mathcal{F}_{t}$.

2.1. Production of Goods. A finite set $I$ of firms produce and sell a set $K$ of different goods at times $0,1, \ldots, T-1$. Each firm $i \in I$ has access to a set $J^{i, k}$ of different technologies to produce good $k \in K$, that are sources of emissions (e.g. greenhouse gases ). Each technology $j \in J^{i, k}$ is characterized by:

- a marginal cost $\widetilde{C}_{t}^{i, j, k}$ of producing one unit of good $k$ at time $t$;

- an emission factor $e^{i, j, k}$ measuring the volume of pollutants emitted per unit of good $k$ produced by firm $i$ with technology $j$;

- a production capacity $\kappa^{i, j, k}$. 
For the sake of notation we introduce the index sets

$$
\begin{aligned}
M_{i} & =\left\{(j, k): k \in K, j \in J^{i, k}\right\}, \quad i \in I, \\
M & =\left\{(i, j, k): i \in I, k \in K, j \in J^{i, k}\right\} .
\end{aligned}
$$

In this paper, our main example of produced good is electricity. We make the assumption that the production costs are non-negative, adapted and integrable processes.

At each time $0 \leq t \leq T-1$, firm $i \in I$ decides to produce throughout the period $[t, t+1)$ the amount $\xi_{t}^{i, \bar{j}, k}$ of good $k \in K$, using the technology $j \in J^{i, k}$. Since the choice of the production level $\xi_{t}^{i, j, k}$ is based only on present and past observations, the processes $\xi^{i, j, k}$ are supposed adapted and, since production cannot exceed capacity, we require that the inequalities

$$
0 \leq \xi_{t}^{i, j, k} \leq \kappa^{i, j, k}, \quad i \in I, k \in K, j \in J^{i, k}, t=0,1, \cdots, T-1,
$$

hold almost surely. Our market is driven by an exogenous and inelastic demand for goods. Since electricity production is a significant proportion of the emissions covered by the existing schemes, this inelasticity assumption is reasonable. We denote by $D_{t}^{k}$ the demand at time $t$ for good $k \in K$. This demand process is supposed to be adapted to the filtration $\left\{\mathcal{F}_{t}\right\}_{t}$. For each good $k \in K$, we assume that the demand is always smaller than the total production capacity for this good, namely that:

$$
0 \leq D_{t}^{k} \leq \sum_{i \in I} \sum_{j \in J^{i, k}} \kappa^{i, j, k} \quad \text { almost surely, } k \in K .
$$

This assumption is a natural extension of the assumption of inelasticity of the demand as it will conveniently discard issues such as blackouts which would only be a distraction given the purposes of the paper.

2.2. Emission Trading. We denote by $\pi \in[0, \infty)$ the penalty per unit of pollutant. For example, in the original design of the European Union Emissions Trading Scheme (EU-ETS) $\pi$ was set to $40 €$ per metric ton of Carbon Dioxyde equivalent $\left(\mathrm{tCO}_{2} \mathrm{e}\right)$. For each firm, the net cumulative emission is the amount of emissions which have not been offset by allowances at the end of the compliance period. It is computed at time $T$ as the difference between the total amount of pollutants emitted over the entire period $[0, T]$ minus the number of allowances held by the firm at time $T$ and redeemed for the purpose of emissions abatement. The net cumulative emission is this difference whenever positive, and 0 otherwise.

For the sake of simplicity we assume that the entire period $[0, T]$ corresponds to one simple compliance period. In particular, at maturity $T$, all the firms have to cover their emissions by allowances or pay a penalty. Moreover, certificates become worthless if not used as we do not allow banking from one phase to the next. So in this economy, operators of installations that emit pollutants will have two fundamental choices in order to avoid unwanted penalties: reduce emissions by producing with cleaner technologies or buy allowances.

At time 0 , each firm $i \in I$ is given an initial endowment of $\Lambda_{0}^{i}$ allowances. So if it were to hold on to this initial allowance endowment until the end, it would be able to offset up to $\Lambda_{0}^{i}$ units of emissions, and start paying only if its actual cumulative emissions exceed that cap level. This is the cap part of a cap-and-trade scheme. Depending upon their views on the demands for the various products and their risk appetites, firms may choose production schedules leading to cumulative emissions in 
excess of their caps. In order to offset expected penalties, they may engage in buying allowances from firms which expect to meet demand with less emissions than their own cap. This is the trade part of a cap-and-trade schemes.

REMARK 1. A first generalization of the above allowance distribution scheme is to reward the firms with allocations $\Lambda_{t}^{i}$ at each time $t=0,1, \cdots, T-1$. Even though modeling EU-ETS would only require one initial (deterministic) allocation $\Lambda_{0}^{i}$ for each firm, we shall assume that the distribution of pollution permits is given by adapted stochastic processes $\left\{\Lambda_{t}^{i}\right\}_{t=0,1, \cdots, T-1}$. Indeed, all the theoretical results proven in the paper hold for these more general permit allocation processes since existence, uniqueness and characterization of the equilibrium price processes depend only upon the total number of emission permits issued during the compliance period, not on the way the permits are distributed over time and among the various economic agents.

However as we will demonstrate, the statistical properties of social costs and windfall profits depend strongly on the way permits are allocated. The challenge faced by policy makers is to optimally design these allocation schemes to minimize social costs while satisfying emissions reduction targets, controlling producers windfall profits and setting incentives for the development of cleaner production technologies. We shall concentrate on these issues in Sections 6 and $\%$.

Allowances are physical in nature, since they are certificates which can be redeemed at time $T$ to offset measured emissions. But, because of trading, these certificates change hands at each time $t=0,1, \cdots, T$, and they become financial instruments. However in general the allocation of allowances does not take place at a single timepoint 0. For example, in EU ETS, allowances are allocated in March each year, while the 5 year compliance period starts in January. Therefore a significant amount of allowances are traded via forward contracts. Because compliance takes place at time $T$, and only at that time, we will restrict ourselves to the situation where trading of emission allowances is done via forward contracts settled at time $T$.

REMARK 2. Because compliance takes place at time $T$, a simple no-arbitrage argument implies that the forward and spot allowance prices differ only by a discounting factor, such that trading allowances or forwards gives the same expected discounted payoff at time $T$. Therefore under the equilibrium definition that will be introduced in Section 3, considering only forward trading yields no loss of generality. Moreover allowing trading in forward contracts in our model provides a more flexible setting: it is more general than considering only spot trading, since it allows for trading pollution permits even before these allowances are issued and allocated. This turns out to be an important feature when dealing with general allocation schemes.

We denote by $A_{t}$ the price at time $t$ of a forward contract guaranteeing delivery of one allowance certificate at maturity $T$. The terminology price at time $t$ is misleading as there is no exchange of funds at time $t$. $A_{t}$ is better seen as a strike than a price in the sense that it is the price (in time $T$ currency) at which the buyer at time $t$ of the forward contract agrees to purchase the allowance certificate at time $T$.

Each firm can take positions on the forward market, and we denote by $\theta_{t}^{i}$ the number of forward contracts held by firm $i$ at the beginning of the time interval $[t, t+1)$. As usual, $\theta_{t}^{i}>0$ when the firm is long and $\theta_{t}^{i}<0$ when it is short. We define a trading strategy of firm $i$ as an adapted process $\left\{\theta_{t}^{i}\right\}_{t=0, \cdots, T}$. If we denote by $f_{t}^{i}$ the quantity of forward contracts bought or sold at time $t$ and throughout the period $[t, t+1), f^{i}$ being an adapted process, the position at time $t$ verifies: $\theta_{t+1}^{i}=\theta_{t}^{i}+f_{t}^{i}$. The net cash position resulting from this trading strategy, leading to a net position 
of $\theta_{T}^{i}$ contracts at time $T$, is:

$$
R_{T}^{A}(\theta):=-\sum_{t=0}^{T} f_{t}^{i} A_{t}=\sum_{t=0}^{T-1} \theta_{t}^{i}\left(A_{t+1}-A_{t}\right)-\theta_{T}^{i} A_{T} .
$$

We here make the assumption that allowances can be traded until time $T$, whereas production of goods is decided at time $t$ for the whole period $[t, t+1)$, so that the last production decision occurs at time $T-1$. This assumption is reasonable since production of good is a less flexible process than trading.

2.3. Profits. As we argued earlier, it is natural to work with $T$-forward allowance contracts because compliance takes place at time $T$. By consistency, it is convenient to express all cash flows, position values, firm wealth, and good values in time $T$-currency. As a side fringe benefit, this will avoid discounting in the computations to come. So we use for numéraire the price $B_{t}(T)$ at time $t$ of a Treasury (i.e. non defaultable) zero coupon bond maturing at $T$. We denote by $\left\{\tilde{S}_{t}^{k}\right\}_{t=0,1, \cdots, T}$ the adapted spot price process of good $k \in K$, and according to the convention stated above, we shall find it convenient to work at each time $t$ with the $T$-forward price

$$
S_{t}^{k}=\tilde{S}_{t}^{k} / B_{t}(T)
$$

and we skip the dependence in $T$ from the notation of the $T$-forward price as $T$ is the only maturity we are considering.

Hence, a cash flow $X_{t}$ at time $t$ is equivalently valued as a cash flow $X_{t} / B_{t}(T)$ at maturity $T$. So if firm $i$ follows the production policy $\xi^{i}=\left\{\left(\xi_{t}^{i, j, k}\right)_{k \in K} j \in J^{i, k}\right\}_{t=0}^{T-1}$ its instantaneous revenues at time $t$ from goods production is given by

$$
\sum_{(j, k) \in M_{i}}\left(\tilde{S}_{t}^{k}-\widetilde{C}_{t}^{i, j, k}\right) \xi_{t}^{i, j, k}
$$

and its time $T$-forward value is given by:

$$
\sum_{(j, k) \in M_{i}}\left(S_{t}^{k}-C_{t}^{i, j, k}\right) \xi_{t}^{i, j, k}
$$

provided we set $C_{t}^{i, j, k}=\widetilde{C}_{t}^{i, j, k} / B_{t}(T)$. The total net gains from producing and selling goods are thus:

$$
\sum_{t=0}^{T-1} \sum_{(j, k) \in M_{i}}\left(S_{t}^{k}-C_{t}^{i, j, k}\right) \xi_{t}^{i, j, k}
$$

In order to hedge their production decisions, firms trade on the emissions market by adjusting their forward positions in allowances. In addition, at maturity $T$, each firm $i$ redeems allowances to cover its emissions and/or pay a penalty. Let

$$
\Pi^{i}\left(\xi^{i}\right):=\sum_{t=0}^{T-1} \sum_{(j, k) \in M_{i}} e^{i, j, k} \xi_{t}^{i, j, k}
$$

be the actual cumulative emissions of firm $i$ when it uses production strategy $\xi^{i}$. We also suppose that there exists another source of emissions on which firm $i$ has 
no control, denoted $\Delta^{i}$, and supposed to be an $\mathcal{F}_{T}$-measurable random variable. If we think of electricity as one of the produced goods for example, the presence of this uncontrolled source of emissions can easily be explained. Usually electricity producers are required to hold a reserve margin in order to respond to short time demand changes and to protect against sudden outages or unexpectedly rapid ramps in demand. When scheduling their plants it is not yet known how much of this reserve margin will be used. Therefore in most markets there is an uncertainty on the exact emission level when a production decision is made. Alternatively, we can see $\Delta^{i}$ as a sink of emissions, accounting for example for the credits gained from Clean Development Mechanisms or Joint Implementation mechanisms. In this case it can take negative values. In a first reading $\Delta^{i}$ can be thought of as being 0 for the sake of simplicity. We shall see later in the paper that its presence helps characterizing the equilibrium of the economy and that it is a useful tool for modeling several variations of the model. Introducing the net amount $\Gamma^{i}$ of allowances that producer $i \in I$ can use to offset the scheduled emissions by

$$
\Gamma^{i}=\Delta^{i}-\sum_{t=0}^{T-1} \Lambda_{t}^{i}
$$

the total penalty paid by firm $i$ at time $T$ is:

$$
\pi\left(\Gamma^{i}+\Pi^{i}\left(\xi^{i}\right)-\theta_{T}^{i}\right)^{+} .
$$

Combining (2.4) and (2.7) together with (2.3), we obtain the expression for the terminal wealth (profits and losses at time $T$ ) of firm $i$ :

$$
\begin{aligned}
L^{A, S, i}\left(\theta^{i}, \xi^{i}\right):=\sum_{t=0}^{T-1} & \sum_{(j, k) \in M_{i}}\left(S_{t}^{k}-C_{t}^{i, j, k}\right) \xi_{t}^{i, j, k} \\
& +\sum_{t=0}^{T-1} \theta_{t}^{i}\left(A_{t+1}-A_{t}\right)-\theta_{T}^{i} A_{T} \\
& \quad \pi\left(\Gamma^{i}+\Pi^{i}\left(\xi^{i}\right)-\theta_{T}^{i}\right)^{+} .
\end{aligned}
$$

To emphasize the mathematical technicalities of the model, we underline the fact that demands and production costs change with time in a stochastic manner. The statistical properties of these processes are given exogenously, and are known at time 0 by all firms. Moreover, we always assume that these processes satisfy the constraints (2.1) and (2.2) almost surely. Agents adjust their production and trading strategies in a non-anticipative manner to their observations of the fluctuations in demand and production costs. In turn, the production and trading strategies $\xi^{i}$ and $\theta^{i}$ become respectively adapted stochastic processes on the stochastic base of the demand and production costs.

3. Market Equilibrium. In this section, we follow the common apprehension that a realistic market state is described by prices which correspond to a so-called market equilibrium, a situation, where the demand for each product is covered, all financial positions are in the zero net supply, and each firm is satisfied by its own strategy. We define such an equilibrium and provide necessary conditions for its existence. 
3.1. Definition of Equilibrium. For any $1 \leq p \leq \infty$ and for any normed vector space $F$, we introduce the following space of adapted processes:

$$
\mathcal{L}_{t}^{p}(F):=\left\{\left(X_{s}\right)_{s=0}^{t} ; F \text {-valued, }\left\|X_{s}\right\| \in L^{p}\left(\mathcal{F}_{s}\right), s=0, \ldots, t\right\} .
$$

We also introduce the spaces of admissible production strategies:

$$
\begin{aligned}
\mathcal{U}^{i} & :=\left\{\left(\xi_{t}^{i}\right)_{t=0}^{T-1} \in \mathcal{L}_{T-1}^{\infty}\left(\mathbb{R}^{M_{i}}\right) ; 0 \leq \xi_{t}^{i, j, k} \leq \kappa^{i, j, k}, \quad t=0, \ldots, T-1\right\}, \\
\mathcal{U} & :=\left\{\xi \in \prod_{i \in I} \mathcal{U}^{i} ; \sum_{i \in I} \sum_{j \in J^{i, k}} \xi_{t}^{i, j, k} \geq D_{t}^{k}, k \in K, \quad t=0, \cdots, T-1\right\}
\end{aligned}
$$

and the spaces of admissible trading strategies:

$$
\begin{aligned}
\mathcal{V}^{i}(A) & :=\left\{\left(\theta_{t}^{i}\right)_{t=1}^{T+1}, \text { adapted, }\left\|R_{T}^{A}\left(\theta^{i}\right)\right\| \in L^{1}\left(\mathcal{F}_{T}\right)\right\} \\
\mathcal{V}(A) & :=\prod_{i \in I} \mathcal{V}^{i}(A) .
\end{aligned}
$$

In order to avoid problems with existence of expected values in (2.8), we suppose that allowance demand and production costs are integrable:

ASSUMPTION 1.

$$
\Gamma^{i} \in L^{1}, \quad\left\{C_{t}^{i}=\left(C_{t}^{i, j, k}\right)_{(j, k) \in M_{i}}\right\}_{t=0}^{T-1} \in \mathcal{L}_{T-1}^{1}\left(\mathbb{R}^{M_{i}}\right) \quad i \in I,
$$

In what follows, we also use a technical assumption on the nature of the uncontrolled emissions. Even though this assumption is not needed for most of the equilibrium existence results, it will help us characterize the prices in equilibrium by ruling out pathological situations. This technical assumption states that up until the end of the compliance period, there is always uncertainty about the expected pollution level due to unpredictable events as described in Section 2.3 in the sense that conditionally on the information available at time $T-1$, the sum of all the $\Gamma^{i}$, s has a continuous distribution. More precisely, we shall assume that

ASSUMPTION 2. the $\mathcal{F}_{T-1}$-conditional distribution of $\sum_{i \in I} \Delta^{i}$ possesses almost surely no point mass, or equivalently, for all $\mathcal{F}_{T-1}$-measurable random variables $Z$

$$
\mathbb{P}\left\{\sum_{i \in I} \Delta^{i}=Z\right\}=0
$$

As we already pointed out, this technical assumption will help us refine the statements of some of the results leading to the equilibriums.

Following the intuition that given price processes $A=\left\{A_{t}\right\}_{t=0}^{T}$ and $S=\left\{\left(S_{t}^{k}\right)_{k \in K}\right\}_{t=0}^{T-1}$ each firm aims at increasing its own wealth by maximizing

$$
\left(\theta^{i}, \xi^{i}\right) \mapsto \mathbb{E}\left[L^{A, S, i}\left(\theta^{i}, \xi^{i}\right)\right],
$$

over its admissible investment and production strategies, we are led to define equilibrium in the following way:

Definition 1. The pair of price processes $\left(A^{*}, S^{*}\right) \in \mathcal{L}_{T}^{1}(\mathbb{R}) \times \mathcal{L}_{T-1}^{1}\left(\mathbb{R}^{|K|}\right)$ are an equilibrium of the market if for each $i \in I$ there exists $\left(\theta^{* i}, \xi^{* i}\right) \in \mathcal{V}^{i}\left(A^{*}\right) \times \mathcal{U}^{i}$ such 
that:

(i) All financial positions are in zero net supply, i.e.

$$
\sum_{i \in I} \theta_{t}^{* i}=0, \quad t=0, \ldots, T
$$

(ii) Supply meets demand for each good

$$
\sum_{i \in I} \sum_{j \in J^{i, k}} \xi_{t}^{i, j, k}=D_{t}^{k}, \quad k \in K, \quad t=0, \ldots, T-1
$$

(iii) Each firm $i \in I$ is satisfied by its own strategy in the sense that

$$
\mathbb{E}\left[L^{A^{*}, S^{*}, i}\left(\theta^{* i}, \xi^{* i}\right)\right] \geq \mathbb{E}\left[L^{A^{*}, S^{*}, i}\left(\theta^{i}, \xi^{i}\right)\right] \quad \text { for all }\left(\theta^{i}, \xi^{i}\right) \in \mathcal{V}^{i}\left(A^{*}\right) \times \mathcal{U}^{i}
$$

3.2. Equilibrium in the Business As Usual Scenario . When the penalty $\pi$ is equal to zero, an equilibrium should correspond to the Business As Usual scenario. As we explain below, it is characterized by the classical merit order production strategy. At time $t$ and for each good $k$, all the production means of the economy are ranked by increasing production costs $C_{t}^{i, j, k}$. Demand is met by producing from the cheapest production means and good $k$ 's equilibrium spot price is the marginal cost of production of the most expensive production means used to meet demand $D_{t}^{k}$.

More precisely, if $\left(A^{*}, S^{*}\right)$ is an equilibrium, the optimization problem of firm $i$ is

$$
\sup _{\left(\theta^{i}, \xi^{i}\right) \in \mathcal{V}^{i}\left(A^{*}\right) \times \mathcal{U}^{i}} \mathbb{E}\left[\sum_{t=0}^{T-1} \sum_{(j, k) \in M_{i}}\left(S_{t}^{k}-C_{t}^{i, j, k}\right) \xi_{t}^{i, j, k}+\sum_{t=0}^{T-1} \theta_{t}^{i}\left(A_{t+1}-A_{t}\right)-\theta_{T}^{i} A_{T}\right] .
$$

Trading and production strategies are thus decoupled from each other and we are left with a classical competitive equilibrium problem where each firm maximizes

$$
\sup _{\xi^{i} \in \mathcal{U}^{i}} \mathbb{E}\left[\sum_{t=0}^{T-1} \sum_{(j, k) \in M_{i}}\left(S_{t}^{k}-C_{t}^{i, j, k}\right) \xi_{t}^{i, j, k}\right]
$$

and the equilibrium prices $S^{*}$ are set so that supply meets demand. The solution of this equilibrium problem is given by the following linear program for each good $k \in K$ :

$$
\begin{aligned}
\left(\left(\xi_{t}^{* i, j, k}\right)_{j \in J^{i, k}}\right)_{i \in I}=\operatorname{argmax}_{\left(\left(\xi_{t}^{i, j, k}\right)_{j \in J^{i, k}}\right)_{i \in I}} & \sum_{i \in I} \sum_{j \in J^{i, k}}-C_{t}^{i, j, k} \xi_{t}^{i, j, k} \\
\text { s.t. } & \sum_{i \in I} \sum_{j \in J^{i, k}} \xi_{t}^{i, j, k}=D_{t}^{k} \\
& \xi_{t}^{i, j, k} \leq \kappa^{i, j, k} \quad \text { for } i \in I, j \in J^{i, k} \\
& \xi_{t}^{i, j, k} \geq 0 \quad \text { for } i \in I, j \in J^{i, k} .
\end{aligned}
$$

for all times $t$, and the associated equilibrium prices are

$$
S_{t}^{* k}=\max _{i \in I, j \in J^{i, k}}\left(C_{t}^{i, j, k}\right) 1_{\left\{\xi_{t}^{* i, j, k}>0\right\}}
$$


This is exactly the merit order pricing mechanism of electricity that can be observed in most deregulated electricity markets without emission trading scheme. Conversely, it is easily seen that the above prices together with the above strategies define an equilibrium. In Section 4 we will see that even under an emission trading scheme the dispatching of production among producers is still a merit order-like dispatching with costs adjusted to take into account the mark-to-market value of emissions.

3.3. Necessary Conditions for the Existence of an Equilibrium. Before turning to the full characterization of the equilibriums, we present some necessary conditions that will provide interesting insight.

Proposition 3.1 (Necessary Conditions). Let $\left(A^{*}, S^{*}\right)$ be an equilibrium and $\left(\theta^{*}, \xi^{*}\right)$ an associated optimal strategies, then following conditions hold:

(i) Then the allowance price $A^{*}$ is a bounded martingale with values in $[0, \pi]$ such that

$$
\left\{A_{T}^{*}=0\right\} \supseteq\left\{\Gamma+\Pi\left(\xi^{*}\right)<0\right\}, \quad\left\{A_{T}^{*}=\pi\right\} \supseteq\left\{\Gamma+\Pi\left(\xi^{*}\right)>0\right\}
$$

up to sets of probability zero.

(ii) If moreover Assumption 2 holds, then it follows that $A^{*}$ is almost surely given by

$$
A_{t}^{*}=\pi \mathbb{E}\left[\mathbf{1}_{\left.\left\{\Gamma+\Pi\left(\xi^{*}\right)\right) \geq 0\right\}} \mid \mathcal{F}_{t}\right]
$$

for all $t=0, \ldots, T$.

(iii) The spot prices $S^{* k}$ and the optimal production strategy $\xi^{* i}$ correspond to a merit order-type equilibrium with adjusted costs $C_{t}^{i, j, k}+e^{i, j, k} A_{t}^{*}$.

Proof. First let us show that $A^{*}$ has to be a martingale. This is seen as follows: if not, there exists a time $t$ and a set $\mathcal{A} \in \mathcal{F}_{t}$ of non-zero probability such that $\mathbb{E}_{t}\left[A_{t+1}^{*} \mathbf{1}_{\mathcal{A}}\right]>\mathbf{1}_{\mathcal{A}} A_{t}^{*}$ (resp. $<$ ). Then for each agent $i \in I$ the trading strategy given by $\bar{\theta}_{s}^{i}=\theta_{s}^{* i}$ for all $s \neq t$ and $\bar{\theta}_{t}^{i}=\theta_{t}^{* i}+1_{\mathcal{A}}\left(\operatorname{resp} \bar{\theta}_{t}^{i}=\theta_{t}^{* i}-1_{\mathcal{A}}\right)$ outperforms the strategy $\theta^{* i}$, contradicting the third property of an equilibrium.

To prove (3.11) notice that according to the definition of the equilibrium, $\theta_{T}^{* i}(\omega)$ coincides for almost all $\omega \in \Omega$ with the maximizer of

$$
z \rightarrow-A_{T}^{*}(\omega) z-\pi\left(\Gamma^{i}(\omega)+\Pi^{i}\left(\xi^{* i}\right)(\omega)-z\right)^{+} .
$$

As a consequence, we obtain $A_{T}^{*} \in[0, \pi]$ almost surely, since if $A_{T}^{*}(\omega) \notin[0, \pi]$ then there exists no maximizer to (3.13). Further, observe that if $A_{T}^{*}(\omega) \in(0, \pi]$ then the maximizer is less than or equal to $\Gamma^{i}(\omega)+\Pi^{i}\left(\xi^{* i}\right)(\omega)$ and if $A_{T}^{*}(\omega) \in[0, \pi)$ then the maximizer is greater than or equal to $\Gamma^{i}(\omega)+\Pi^{i}\left(\xi^{* i}\right)(\omega)$. This holds for each $i$ hence following inclusions are satisfied almost surely

$$
\begin{aligned}
& \left\{A_{T}^{*} \in(0, \pi]\right\} \subseteq \cap_{i \in I}\left\{\theta_{T}^{* i} \leq \Gamma^{i}+\Pi^{i}\left(\xi^{*}\right)\right\} \subseteq\left\{\sum_{i \in I} \theta_{T}^{* i} \leq \Gamma+\Pi\left(\xi^{*}\right)\right\} \\
& \left\{A_{T}^{*} \in[0, \pi)\right\} \subseteq \cap_{i \in I}\left\{\theta_{T}^{* i} \geq \Gamma^{i}+\Pi^{i}\left(\xi^{*}\right)\right\} \subseteq\left\{\sum_{i \in I} \theta_{T}^{* i} \geq \Gamma+\Pi\left(\xi^{*}\right)\right\} .
\end{aligned}
$$

That is

$$
\begin{aligned}
& \left\{A_{T}^{*} \in(0, \pi]\right\} \cap\left\{\Gamma+\Pi\left(\xi^{*}\right)<0\right\} \subseteq\left\{\sum_{i \in I} \theta_{T}^{* i}<0\right\} \\
& \left\{A_{T}^{*} \in[0, \pi)\right\} \cap\left\{\Gamma+\Pi\left(\xi^{*}\right)>0\right\} \subseteq\left\{\sum_{i \in I} \theta_{T}^{* i}>0\right\} .
\end{aligned}
$$


Observe that due to the first equilibrium condition $\sum_{i \in I} \theta_{T}^{* i}=0$, the events on the right hand sides of (3.16) and (3.17) are sets of probability zero which shows that the inclusions (3.11) hold almost surely. Condition (ii) is a direct consequence of (i) and Assumption 2.

Finally, the optimization problem of agent $i$ can be written as:

$$
\begin{aligned}
\sup _{\theta^{i} \in \mathcal{U}^{i}} \mathbb{E}\left[\sum_{t=0}^{T-1} \sum_{(j, k) \in M_{i}}\left(S_{t}^{k}-C_{t}^{i, j, k}-e^{i, j, k} A_{T}^{*}\right) \xi_{t}^{i, j, k}\right] \\
=\sup _{\theta^{i} \in \mathcal{U}^{i}} \mathbb{E}\left[\sum_{t=0}^{T-1} \sum_{(j, k) \in M_{i}}\left(S_{t}^{k}-C_{t}^{i, j, k}-e^{i, j, k} A_{t}^{*}\right) \xi_{t}^{i, j, k}\right]
\end{aligned}
$$

thanks to the martingale property of $A^{*}$. Comparing the above optimization problem with (3.8), we observe that the equilibrium can be seen as a competitive production equilibrium with adjusted $\operatorname{costs} C_{t}^{i, j, k}+e^{i, j, k} A_{t}^{*}$.

This concludes the proof.

$\square$

The above results provide a better understanding of what a potential equilibrium should be. The allowance price must always be in $[0, \pi]$, which is very intuitive since buying an extra allowance at time $t$ will result in a gain of at most $\pi$ at time $T$. As highlighted in the previous section, the equilibrium in the BAU scenario can be related to a global cost minimization problem. We shall see in the next section that the equilibrium in the presence of a trading scheme enjoys the property of social optimality in the sense that any equilibrium corresponds to the solution of a certain global optimization problem, where the total pollution is reduced at minimal overall costs. We call this optimization problem the representative firm problem. Beyond the economic interpretation of social-optimality, the importance of the global optimization problem is that its solution helps calculate the allowance prices in equilibrium. We now explore this connection in detail.

4. Equilibrium and Global Optimality. In this section, we show rigorously the existence of an equilibrium as defined in Definition 1. We do so by re-framing the problem as an equivalent global optimization problem involving a hypothetical informed central planner (which we call a representative agent). We prove the equivalence of the two approaches, and as a by-product of the necessary condition proven in the previous section, we derive the uniqueness of the allowance price process.

4.1. The Representative Agent Problem . For each admissible production strategy $\xi=\left\{\xi^{i}\right\}_{i \in I} \in \mathcal{U}$, the overall production costs are defined as

$$
C(\xi):=\sum_{t=0}^{T-1} \sum_{(i, j, k) \in M} \xi_{t}^{i, j, k} C_{t}^{i, j, k} .
$$

and the overall cumulated emissions as

$$
\Pi(\xi):=\sum_{t=0}^{T-1} \sum_{(i, j, k) \in M} e^{i, j, k} \xi_{t}^{i, j, k} .
$$


Using the notation

$$
\Gamma:=\sum_{i \in I} \Gamma^{i}
$$

for the aggregate uncontrolled emissions and allowance endowments, the total costs from production and penalty payments can be defined as

$$
G(\xi):=C(\xi)+\pi(\Gamma+\Pi(\xi))^{+}, \quad \xi \in \mathcal{U}
$$

We introduce the global optimization problem

$$
\inf _{\xi \in \mathcal{U}} \mathbb{E}[G(\xi)]
$$

which corresponds to the objective of an informed central planner trying to minimize overall expected costs. Recall that $\xi$ is admissible if $\xi \in \mathcal{U}$, i.e. if the demand is met and the production constraints are satisfied. The reason for the introduction of this global optimization problem is contained in the second necessary condition for the existence of equilibrium.

Proposition 4.1. If $\left(A^{*}, S^{*}\right)$ is an equilibrium with associated strategies $\left(\theta^{*}, \xi^{*}\right)$, then $\xi^{*}$ is a solution of the global optimization problem (4.3).

Proof. Obviously, it suffices to show that

$$
E\left(G\left(\xi^{*}\right)\right) \leq E(G(\xi)) \text { for all } \xi \in \mathcal{U}
$$

In order to do so we notice that:

$$
\begin{aligned}
& \sum_{i \in I} \mathbb{E}\left[L^{A^{*}, S^{*}, i}\left(\theta^{* i}, \xi^{* i}\right)\right]=\mathbb{E}\left[\sum_{t=0}^{T-1} \sum_{i, j, k \in M}\left(S_{t}^{* k}-C_{t}^{i, j, k}\right) \xi_{t}^{* i, j, k}\right. \\
&+\sum_{t=0}^{T-1}\left(\sum_{i \in I} \theta_{t}^{* i}\right)\left(A_{t+1}^{*}-A_{t}^{*}\right)-\left(\sum_{i \in I} \theta_{T}^{* i}\right) A_{T}^{*} \\
&\left.-\pi \sum_{i \in I}\left(\Gamma^{i}+\Pi^{i}\left(\xi^{* i}\right)-\theta_{T}^{* i}\right)^{+}\right] \\
&=\mathbb{E}\left[\sum_{t=0}^{T-1} \sum_{k \in K} S_{t}^{* k}\left(\sum_{i \in I} \sum_{j \in J^{i, k}} \xi_{t}^{* i, j, k}\right)-C\left(\xi^{*}\right)\right. \\
&\left.\quad-\pi \sum_{i \in I}\left(\Gamma^{i}+\Pi^{i}\left(\xi^{* i}\right)-\theta_{T}^{* i}\right)^{+}\right]
\end{aligned}
$$

where we used the fact that in equilibrium, $\sum_{i \in I} \theta_{t}^{* i}=0$ holds for all $t=0, \ldots, T$ due to condition (i) of Definition 1. Next we use the convexity inequality

$$
\sum_{i \in I} x_{i}^{+} \geq\left(\sum_{i \in I} x_{i}\right)^{+}
$$


and once more the fact that the financial positions are in zero net supply to conclude that

$$
\begin{aligned}
& \sum_{i \in I} \mathbb{E}\left[L^{A^{*}, S^{*}, i}\left(\theta^{* i}, \xi^{* i}\right)\right] \leq \sum_{t=0}^{T-1} \sum_{k \in K} \mathbb{E}\left[S_{t}^{* k} D_{t}^{k}\right]-\mathbb{E}\left[C\left(\xi^{*}\right)\right] \\
&-\pi \mathbb{E}\left[\left(\sum_{i \in I} \Gamma^{i}+\sum_{i \in I} \Pi^{i}\left(\xi^{* i}\right)\right)^{+}\right] \\
&=\sum_{t=0}^{T-1} \sum_{k \in K} \mathbb{E}\left[S_{t}^{* k} D_{t}^{k}\right]-\mathbb{E}\left[C\left(\xi^{*}\right)\right]-\pi \mathbb{E}\left[\left(\Gamma+\Pi\left(\xi^{*}\right)\right)^{+}\right] \\
&=\sum_{t=0}^{T-1} \sum_{k \in K} \mathbb{E}\left[S_{t}^{* k} D_{t}^{k}\right]-\mathbb{E}\left[G\left(\xi^{*}\right)\right] .
\end{aligned}
$$

Now, for each $\xi \in \mathcal{U}$ we define $\theta(\xi)$ as

$$
\begin{aligned}
\theta_{t}^{i}(\xi) & =0 \quad \text { for all } i=1, \ldots, N, t=0, \ldots, T-1, \\
\theta_{T}^{i}(\xi) & =\Gamma^{i}+\Pi^{i}\left(\xi^{i}\right)-\frac{\Gamma+\Pi(\xi)}{|I|} .
\end{aligned}
$$

Repeating the above argument for $(\theta(\xi), \xi)$ yields

$$
\sum_{i \in I} \mathbb{E}\left[L^{A^{*}, S^{*}, i}\left(\theta^{i}(\xi), \xi^{i}\right)\right]=\sum_{t, k} \mathbb{E}\left[S^{* k}{ }_{t} D_{t}^{k}\right]-\mathbb{E}[G(\xi)] .
$$

Applying the third property (each agent is satisfied with its own strategy) of the $\left(A^{*}, S^{*}\right)$ equilibrium to the optimal investment and production strategies $\left(\theta^{* i}, \xi^{* i}\right)$ and $\left(\theta^{i}(\xi), \xi^{i}\right)$ yields

$$
\begin{aligned}
\mathbb{E}\left[G\left(\xi^{*}\right)\right] & \leq \sum_{t, k} \mathbb{E}\left[S_{t}^{* k} D_{t}^{k}\right]-\sum_{i \in I} \mathbb{E}\left[L^{A^{*}, S^{*}, i}\left(\theta^{* i}, \xi^{* i}\right)\right] \\
& \leq \sum_{t, k} \mathbb{E}\left[S_{t}^{* k} D_{t}^{k}\right]-\sum_{i \in I} \mathbb{E}\left[L^{A^{*}, S^{*}, i}\left(\theta^{i}(\xi), \xi\right)\right]=\mathbb{E}[G(\xi)] .
\end{aligned}
$$

This holds for all $\xi \in \mathcal{U}$ completing the proof. $\square$

The existence of an optimal $\bar{\xi}$ for the global optimization problem (4.3) follows from standard functional analytic arguments.

Proposition 4.2. Under Assumption 1, there exists a solution $\bar{\xi} \in \mathcal{U}$ of the global optimal control problem (4.3).

Our proof relies on two simple properties which we state and prove as lemmas for the sake of clarity. First, we note that $\mathcal{L}^{1}:=\prod_{i \in I} \mathcal{L}_{T-1}^{1}\left(\mathbb{R}^{M}\right)$, equipped with the norm

$$
\|X\|=\sum_{t=0}^{T-1} \sum_{(i, j, k) \in M} \mathbb{E}\left[\left|X_{t}^{i, j, k}\right|\right]
$$

is a Banach space with dual $\mathcal{L}^{\infty}:=\prod_{i \in I} \mathcal{L}_{T-1}^{\infty}\left(\mathbb{R}^{M}\right)$, the duality form being given by

$$
\langle X, \xi\rangle:=\sum_{t=0}^{T-1} \sum_{(i, j, k) \in M} \mathbb{E}\left[X_{t}^{i, j, k} \xi_{t}^{i, j, k}\right], \quad X \in \mathcal{L}^{1}, \xi \in \mathcal{L}^{\infty} .
$$


Next, we consider the weak* topology $\sigma\left(\mathcal{L}^{\infty}, \mathcal{L}^{1}\right)$ on $\mathcal{L}^{\infty}$ (see [10]), namely the weakest topology for which all the linear forms

$$
\mathcal{L}^{\infty} \ni \xi \longmapsto\langle X, \xi\rangle \in \mathbb{R},
$$

for $X \in \mathcal{L}^{1}$ are continuous.

LEMMA 4.3. The real valued function

$$
\mathcal{L}^{\infty} \ni \xi \longmapsto \mathbb{E}[G(\xi)]=\mathbb{E}[C(\xi)]+\pi \mathbb{E}\left[(\Gamma+\Pi(\xi))^{+}\right]
$$

is lower semi-continuous for the weak* topology.

Proof. Obviously, the real valued function

$$
\mathcal{L}^{\infty} \ni \xi \longmapsto \mathbb{E}[C(\xi)]
$$

is continuous for the weak* topology since it is of the form $\xi \longmapsto\langle X, \xi\rangle$ for some $X \in \mathcal{L}^{1}$ since $X=C=\left\{C_{t}^{i, j, k}\right\}$ is a fixed element in $\mathcal{L}^{1}$ by assumption. So we only need to prove that the real valued function

$$
\mathcal{L}^{\infty} \ni \xi \longmapsto \mathbb{E}\left[(\Gamma+\Pi(\xi))^{+}\right]
$$

is lower semi-continuous. Using the fact that for any integrable random variable $X$ one has

$$
\mathbb{E}\left[X^{+}\right]=\sup _{0 \leq Y \leq 1} \mathbb{E}[X Y]
$$

one sees that

$$
\mathbb{E}\left[(\Gamma+\Pi(\xi))^{+}\right]=\sup _{0 \leq Y \leq 1}(\mathbb{E}[\Gamma Y]+\mathbb{E}[Y \Pi(\xi)])
$$

and hence that the function (4.8) is the supremum of a family of continuous function since for fixed $Y, \mathbb{E}[\Gamma Y]$ is a constant and $\xi \longmapsto \mathbb{E}[Y \Pi(\xi)]$ is continuous for the weak ${ }^{*}$ topology by the very definition of this topology. Since the supremum of any family of continuous functions is lower semi-continuous, this concludes the proof that (4.7) is lower semi-continuous.

Lemma 4.4. For the convex subset $\mathcal{U}$ of $\mathcal{L}^{\infty}$ it holds that:

(i) $\mathcal{U}$ is norm-closed in $\mathcal{L}^{1}$

(ii) $\mathcal{U}$ is weakly* closed in $\mathcal{L}^{\infty}$.

Proof. (i)If $\left(\xi_{n}\right)_{n \in \mathbb{N}}$ is a sequence in $\mathcal{U}$ converging in $\mathcal{L}^{1}$ to some random variable $\xi$, then $\left(\xi_{t, n}^{i, j, k}\right)_{n \in \mathbb{N}}$ converges in mean for each $(i, j, k) \in M$ and $t=0, \ldots, T-$ 1 , and extracting a subsequence if necessary, one concludes that $\left(\xi_{t, n}^{i, j, k}\right)_{n \in \mathbb{N}}$ and $\left(\sum_{i \in I} \sum_{j \in J^{i, k}} \xi_{t, n}^{i, j, k}\right)_{n \in \mathbb{N}}$ converge almost surely to $\xi_{t}^{i, j, k}$ and $\sum_{i \in I} \sum_{j \in J^{i, k}} \xi_{t}^{i, j, k}$ respectively, showing that the constraints defining $\mathcal{U}$ are satisfied in the limit, implying that $\xi \in \mathcal{U}$.

(ii) Since $\mathcal{U}$ is a convex and a norm-closed subset of $\mathcal{L}^{1}$ it follows from the HahnBanach Theorem that $\mathcal{U}$ is the intersection of halfspaces $H_{\xi, c}=\left\{X \in \mathcal{L}^{1} \mid\langle X, \xi\rangle \leq c\right\}$ with $\xi \in \mathcal{L}^{\infty}$ and $c \in \mathbb{R}$ such that $\mathcal{U} \subseteq H$. Since $\mathcal{L}^{\infty} \subseteq \mathcal{L}^{1}$ it holds for each of these halfspaces $H_{\xi, c}$ that $\xi \in \mathcal{L}^{1}$. Thus we conclude that $H_{\xi, c} \cap \mathcal{L}^{\infty}=\left\{X \in \mathcal{L}^{\infty} \mid\langle X, \xi\rangle \leq c\right\}$ is closed in $\left(\mathcal{L}^{\infty}, \sigma\left(\mathcal{L}^{\infty}, \mathcal{L}^{1}\right)\right)$. Since by definition it holds that $\mathcal{U} \subseteq \mathcal{L}^{\infty}$ it follows that $\mathcal{U}$ is given by the intersection of the sets $H_{\xi, c} \cap \mathcal{L}^{\infty}$. Since any intersection of closed sets is closed we conclude that $\mathcal{U}$ is weakly* closed in $\mathcal{L}^{\infty}$. 
Proof of Proposition 4.2 Since $\mathcal{U}$ is bounded and weakly* closed due to Lemma 4.4, it follows from the Theorem of Banach-Alaoglu that $\mathcal{U}$ is weakly* compact. Lemma 4.3 concludes the proof since any lower semi-continuous function attains its minimum on a compact set.

4.2. Relation with the Original Equilibrium Problem . As a consequence of Assumption 2, for each production policy $\xi \in \mathcal{U}$, no point masses occur in the $\mathcal{F}_{T-1}$-conditional distribution of $\Gamma-\Pi(\xi)$. Hence, for all $t=0, \ldots, T-1$ we have:

$$
\mathbb{P}_{t}(\Gamma+\Pi(\xi) \geq 0)=\mathbb{P}_{t}(\Gamma+\Pi(\xi)>0) .
$$

In the next theorem, we show that the value of the conditional probability in (4.9) characterizes the equilibrium allowance price at time $t$. To prepare for the proof of this result, we first prove a technical lemma.

LEMma 4.5. Let $\bar{\xi}$ be any solution of (4.6) whose existence is guaranteed by Proposition 4.2, then it follows that:

(i) For fixed $t \in\{0, \ldots, T-1\}$ and any $\xi \in \mathcal{U}$ with $\xi_{s}=\bar{\xi}_{s}$ for all $s=0, \ldots, t-1$

$$
E_{t}(G(\xi)) \geq E_{t}(G(\bar{\xi}))
$$

holds almost surely.

(ii) If Assumption 2 is satisfied, then for each $k \in K$ and $i, i^{\prime} \in I, j \in J^{i, k}, j^{\prime} \in J^{i^{\prime}, k}$ it holds that

$$
\begin{aligned}
& \left\{\bar{\xi}_{t}^{i, j, k} \in\left[0, \kappa^{i, j, k}\right)\right\} \cap\left\{\bar{\xi}_{t}^{i^{\prime}, j^{\prime}, k} \in\left(0, \kappa^{i^{\prime}, j^{\prime}, k}\right]\right\} \\
& \quad \subseteq\left\{C_{t}^{i, j, k}+e^{i, j, k} \bar{A}_{t} \geq C_{t}^{i^{\prime}, j^{\prime}, k}+e^{i^{\prime}, j^{\prime}, k} \bar{A}_{t}\right\}
\end{aligned}
$$

for all $t=0, \ldots, T-1$ where $\bar{A}_{t}=\pi \mathbb{P}_{t}(\Gamma+\Pi(\bar{\xi}) \geq 0)$.

Proof. (i) The assertion (4.10) is seen by the following argumentation: On the contrary, one uses the $\mathbb{F}_{t}$-measurable set

$$
\mathcal{O}:=\left\{E_{t}(G(\xi))<E_{t}(G(\bar{\xi})\} \quad \text { of positive measure } \quad P(\mathcal{O})>0,\right.
$$

to outperform $\bar{\xi}$ by $\xi^{\prime}$ as

$$
\xi_{s}^{\prime}=1_{\mathcal{O}} \xi_{s}+1_{\Omega \backslash \mathcal{O}} \bar{\xi}_{s} \text { for all } s=0, \ldots, T-1 .
$$

Note that since $\xi$ and $\xi^{\prime}$ coincide at times $0, \ldots, t-1$, this definition indeed yields an adapted process $\xi^{\prime} \in \mathcal{U}$. With (4.12), we have the decomposition

$$
G\left(\xi^{\prime}\right)=1_{\mathcal{O}} G(\xi)+1_{\Omega \backslash \mathcal{O}} G(\bar{\xi}),
$$

which gives a contradiction to the optimality of $\bar{\xi}$ :

$$
\begin{aligned}
E\left(G\left(\xi^{\prime}\right)\right) & =E\left(E_{t}\left(1_{\mathcal{O}} G(\xi)+1_{\Omega \backslash \mathcal{O}} G(\bar{\xi})\right)\right. \\
& =E\left(1_{\mathcal{O}} E_{t}(G(\xi))+1_{\Omega \backslash \mathcal{O}} E_{t}(G(\bar{\xi}))\right) \\
& <E\left(1_{\mathcal{O}} E_{t}(G(\bar{\xi}))+1_{\Omega \backslash \mathcal{O}} E_{t}(G(\bar{\xi}))\right)=E(G(\bar{\xi})) .
\end{aligned}
$$

(ii) Introduce a deviation from the global optimal strategy $\bar{\xi}$. At time $t$, consider a shift in production of $h_{t}$ units of the good $k \in K$, where the agents $i \in I$ and $i^{\prime} \in I$ increase/decrease their outputs from technologies $j \in \mathcal{J}^{i, k}, j^{\prime} \in \mathcal{J}^{i^{\prime}, k}$ respectively. 
This results in the new policy $\bar{\xi}+\chi$ where $\chi \in \Pi_{i \in I} \mathcal{U}^{i}$ the deviation vanishes at all times with the exception of $t$ and

$$
\chi_{t}^{i, j, k}=h_{t}, \quad \chi_{t}^{i^{\prime}, j^{\prime}, k}=-h_{t},
$$

Consider

$$
D(\bar{\xi}, \lambda)=\frac{E_{t}(G(\bar{\xi}+\lambda \chi))-E_{t}(G(\bar{\xi}))}{\lambda}, \quad \lambda \in(0,1] .
$$

Approaching 0 by $\lambda$ in the countable set $(0,1] \cap \mathbb{Q}$ we obtain by dominated convergence limits for $e^{i, j, k} \leq e^{i^{\prime}, j^{\prime}, k^{\prime}}$ and $e^{i, j, k} \geq e^{i^{\prime}, j^{\prime}, k^{\prime}}$ as

$$
\begin{aligned}
& \lim _{\lambda \rightarrow 0} D(\bar{\xi}, \lambda)=-\left(\left(C_{t}^{i, j, k}-C_{t}^{i^{\prime}, j^{\prime}, k}\right)-\pi P_{t}(\Gamma+\Pi(\bar{\xi})>0)\left(e^{i, j, k}-e^{i^{\prime}, j^{\prime}, k}\right)\right) h_{t} \\
& \lim _{\lambda \rightarrow 0} D(\bar{\xi}, \lambda)=-\left(\left(C_{t}^{i, j, k}-C_{t}^{i^{\prime}, j^{\prime}, k}\right)-\pi P_{t}(\Gamma+\Pi(\bar{\xi}) \geq 0)\left(e^{i, j, k}-e^{i^{\prime}, j^{\prime}, k}\right)\right) h_{t} .
\end{aligned}
$$

That is, with Assumption 2 we obtain the limit as

$$
\lim _{\lambda \rightarrow 0} D(\bar{\xi}, \lambda)=-\left(\left(C_{t}^{i, j, k}-C_{t}^{i^{\prime}, j^{\prime}, k}\right)-\bar{A}_{t}\left(e^{i, j, k}-e^{i^{\prime}, j^{\prime}, k}\right)\right) h_{t} .
$$

Further, if the production shift is given by

$$
h_{t}=\min \left\{\kappa^{i, j, k}-\bar{\xi}_{t}^{i, j, k}, \bar{\xi}_{t}^{i^{\prime}, j^{\prime}, k}\right\}
$$

then $\bar{\xi}+\lambda \chi \in \mathcal{U}$ for all $\lambda \in(0,1] \cap \mathbb{Q}$ which, due to (i), yields

$$
D(\bar{\xi}, \lambda)(\omega) \leq 0, \quad \text { for all } \omega \in \tilde{\Omega} \text { with } P(\tilde{\Omega})=1 .
$$

Passing through the limit $\lambda \downarrow 0$, we obtain with (4.10) and (4.13)

$$
-\left(\left(C_{t}^{i, j, k}-C_{t}^{i^{\prime}, j^{\prime}, k}\right)-P_{t}(\Gamma+\Pi(\bar{\xi})>0)\left(e^{i, j, k}-e^{i^{\prime}, j^{\prime}, k}\right)\right) h_{t} \leq 0
$$

almost surely. Hence the inclusion

$$
\left\{h_{t}>0\right\} \subseteq\left\{-\left(\left(C_{t}^{i, j, k}-C_{t}^{i^{\prime}, j^{\prime}, k}\right)-\bar{A}_{t}\left(e^{i, j, k}-e^{i^{\prime}, j^{\prime}, k}\right)\right) \leq 0\right\}
$$

holds almost surely, which is equivalent to (4.11). $\square$

We can now turn to the main result of this section.

THEOREM 4.6. Under the above assumptions, the following hold:

(i) If $\bar{\xi} \in \mathcal{U}$ is a solution of the global optimization problem (4.3), then the processes $(\bar{A}, \bar{S})$ defined by

$$
\bar{A}_{t}=\pi \mathbb{P}_{t}(\Gamma+\Pi(\bar{\xi}) \geq 0), \quad t=0, \ldots, T
$$

and

$$
\bar{S}_{t}^{k}=\max _{i \in I, j \in J^{i, k}}\left(C_{t}^{i, j, k}+e^{i, j, k} \bar{A}_{t}\right) 1_{\left\{\bar{\xi}_{t}^{i, j, k}>0\right\}}, \quad t=0, \ldots, T-1 k \in K,
$$

is a market equilibrium (in the sense of Definition 1), for which the associated production strategy is $\bar{\xi}$.

(ii) The equilibrium allowance price process is almost surely unique. 
(iii) For each good $k \in K$, the price $\bar{S}^{k}$ is the smallest equilibrium price for good $k$ in the sense that for any other equilibrium price process $S^{* k}$, we have $\bar{S}^{k} \leq S^{* k}$ almost surely.

Proof. (i) We show that $(\bar{A}, \bar{S})$ so defined forms an equilibrium by an explicit construction of firm investment strategies $\bar{\theta}^{i} \in \mathcal{V}^{i}(\bar{A})$ such that $\left(\bar{\theta}^{i}, \bar{\xi}^{i}\right)$ satisfies (3.5), (3.6) and (3.7). Define

$$
\begin{aligned}
\bar{\theta}_{t}^{i} & =0 \quad \text { for all } i=1, \ldots, N, t=1, \ldots, T-1, \\
\bar{\theta}_{T}^{i} & =\Gamma^{i}+\Pi^{i}\left(\bar{\xi}^{i}\right)-\frac{\Gamma+\Pi(\bar{\xi})}{|I|} .
\end{aligned}
$$

Since conditions (3.5) and (3.6) are obviously fulfilled, we focus on (3.7). We first show that $\mathbb{E}\left[L^{\bar{A}, \bar{S}, i}\left(\bar{\theta}^{i}\left(\xi^{i}\right), \xi^{i}\right)\right] \geq \mathbb{E}\left[L^{\bar{A}, \bar{S}, i}\left(\theta^{i}, \xi^{i}\right)\right]$ for all $\left(\theta^{i}, \xi^{i}\right) \in \mathcal{V}^{i}(\bar{A}) \times \mathcal{U}^{i}$, where $\bar{\theta}^{i}\left(\xi^{i}\right)$ is constant equal to 0 until time $T-1$ and

$$
\bar{\theta}_{T}^{i}\left(\xi^{i}\right):=\Gamma^{i}+\Pi^{i}\left(\xi^{i}\right)-\frac{\Gamma+\Pi(\bar{\xi})}{|I|} .
$$

We have:

$$
\begin{aligned}
\mathbb{E}\left[L^{\bar{A}, \bar{S}, i}\left(\theta^{i}, \xi^{i}\right)\right]= & \mathbb{E}\left[\sum_{t=0}^{T-1} \sum_{(j, k) \in M_{i}}\left(\bar{S}_{t}^{k}-C_{t}^{i, j, k}\right) \xi_{t}^{i, j, k}-\theta_{T}^{i} \bar{A}_{T}\right. \\
& \left.-\pi\left(\Gamma^{i}+\Pi^{i}\left(\xi^{i}\right)-\theta_{T}^{i}\right)^{+}\right]
\end{aligned}
$$

since $\bar{A}$ defined by (4.14) is a bounded martingale. For all $\xi^{i} \in \mathcal{U}^{i}$, we show that we can maximize the above quantity by computing the maximum pointwise in $\theta^{i}$ inside the expectation. In view of (4.14), when $\omega \in\{\Gamma+\Pi(\bar{\xi})<0\}$ we have $\omega \in \bar{A}_{T}(\omega)=0$ and the maximum of

$$
z \mapsto-z \bar{A}_{T}(\omega)-\pi\left(\Gamma^{i}(\omega)+\Pi^{i}\left(\xi^{i}\right)(\omega)-z\right)^{+}
$$

is attained on each point $z \in\left[\Gamma^{i}(\omega)+\Pi^{i}\left(\xi^{i}\right)(\omega), \infty\right)$ showing that $\bar{\theta}^{i}\left(\xi^{i}\right)(\omega)$ is a maximizer. On the other hand, when $\omega \in\{\Gamma+\Pi(\bar{\xi}) \geq 0\}$, we have $\bar{A}_{T}(\omega)=\pi$, the maximum of (4.16) is attained on each point $z \in\left(-\infty, \Gamma^{i}(\omega)+\Pi^{i}\left(\xi^{i}\right)(\omega)\right]$, and once again, $\bar{\theta}^{i}\left(\xi^{i}\right)$ is a maximizer. Notice for later reference that in both cases, the value of the maximum of (4.16) is $\mathbb{E}\left[-\left(\Gamma^{i}+\Pi^{i}\left(\xi^{i}\right)\right) \bar{A}_{T}\right]$.

To finish the proof, we prove that $\mathbb{E}\left[L^{\bar{A}, \bar{S}, i}\left(\bar{\theta}^{i}, \bar{\xi}^{i}\right)\right] \geq \mathbb{E}\left[L^{\bar{A}, \bar{S}, i}\left(\bar{\theta}^{i}\left(\xi^{i}\right), \xi^{i}\right)\right]$ for all $\xi^{i} \in \mathcal{U}^{i}$. According to the above computation, we have:

$$
\begin{aligned}
& \mathbb{E}\left[L^{\bar{A}, \bar{S}, i}\left(\bar{\theta}^{i}\left(\xi^{i}\right), \xi^{i}\right)\right] \\
= & \mathbb{E}\left[\sum_{t=0}^{T-1} \sum_{(j, k) \in M_{i}}\left(\bar{S}_{t}^{k}-C_{t}^{i, j, k}\right) \xi_{t}^{i, j, k}-\left(\Gamma^{i}+\Pi^{i}\left(\xi^{i}\right)\right) \bar{A}_{T}\right] \\
= & \mathbb{E}\left[\sum_{t=0}^{T-1} \sum_{(j, k) \in M_{i}}\left(\bar{S}_{t}^{k}-C_{t}^{i, j, k}-e^{i, j, k} \bar{A}_{T}\right) \xi_{t}^{i, j, k}-\Gamma^{i} \bar{A}_{T}\right] \\
= & \mathbb{E}\left[\sum_{t=0}^{T-1} \sum_{(j, k) \in M_{i}}\left(\bar{S}_{t}^{k}-C_{t}^{i, j, k}-e^{i, j, k} \bar{A}_{t}\right) \xi_{t}^{i, j, k}-\Gamma^{i} \bar{A}_{T}\right] .
\end{aligned}
$$


We now show that the following inclusions hold almost surely:

$$
\begin{aligned}
& \left\{\bar{S}_{t}^{k}-C_{t}^{i, j, k}-e^{i, j, k} \bar{A}_{t}>0\right\} \subseteq\left\{\bar{\xi}_{t}^{i, j, k}=\kappa^{i, j, k}\right\}, \\
& \left\{\bar{S}_{t}^{k}-C_{t}^{i, j, k}-e^{i, j, k} \bar{A}_{t}<0\right\} \subseteq\left\{\bar{\xi}_{t}^{i, j, k}=0\right\} .
\end{aligned}
$$

Inclusion (4.19) is a direct consequence of Definition (4.15) of the price process $\bar{S}$. Using this same Definition (4.15) and Lemma 4.5 we see that:

$$
\begin{aligned}
& \left\{\bar{S}_{t}^{k}>C_{t}^{i, j, k}+e^{i, j, k} \bar{A}_{t}\right\} \\
\subseteq & \bigcup_{i^{\prime} \in I, j^{\prime} \in J^{i^{\prime}, k}}\left\{C_{t}^{i^{\prime}, j^{\prime}, k}+e^{i^{\prime}, j^{\prime}, k} \bar{A}_{t}>C_{t}^{i, j, k}+e^{i, j, k} \bar{A}_{t}\right\} \cap\left\{\bar{\xi}^{i^{\prime}, j^{\prime}, k}>0\right\} \\
\subseteq & \bigcup_{i^{\prime} \in I, j^{\prime} \in J^{i^{\prime}, k}}\left(\left\{\bar{\xi}_{t}^{i, j, k}=\kappa^{i, j, k}\right\} \cup\left\{\bar{\xi}_{t}^{i^{\prime}, j^{\prime}, k}=0\right\}\right) \cap\left\{\bar{\xi}^{i^{\prime}, j^{\prime}, k}>0\right\} \\
\subseteq & \left\{\bar{\xi}_{t}^{i, j, k}=\kappa^{i, j, k}\right\} .
\end{aligned}
$$

These inclusions allow us to show that $\mathbb{E}\left[L^{\bar{A}, \bar{S}, i}\left(\bar{\theta}^{i}\left(\xi^{i}\right), \xi^{i}\right)\right] \leq \mathbb{E}\left[L^{\bar{A}, \bar{S}, i}\left(\bar{\theta}^{i}, \bar{\xi}^{i}\right)\right]$, thus completing the proof of (i).

(ii) Proposition 3.1 gives the form of an equilibrium price. Due to Part (i) of Proposition 3.1 and Proposition 4.1 to prove almost sure uniqueness of the allowance price process, it is sufficient to prove that for any two solutions $\hat{\xi}, \tilde{\xi}$ of the global optimization problem (4.3) we have:

$$
\mathbb{P}((\{\Gamma+\Pi(\hat{\xi})>0\} \cap\{\Gamma+\Pi(\tilde{\xi})>0\}) \bigcup(\{\Gamma+\Pi(\hat{\xi})<0\} \cap\{\Gamma+\Pi(\tilde{\xi})<0\}))=1
$$

We know that these production strategies are solution of the global problem (4.3), that we rewrite as a linear programming problem:

$$
\inf _{\substack{\xi \in \mathcal{U}, Z \in L^{1}\left(\mathcal{F}_{T}\right) \\ Z \geq \Gamma+\Pi(\xi)-\theta_{0}, Z \geq 0}} \mathbb{E}[C(\xi)+\pi Z]
$$

Each solution $\left(\xi^{\star}, Z^{\star}\right)$ of $(4.21)$ satisfies

$$
Z^{\star}=\left(\Gamma+\Pi\left(\xi^{\star}\right)\right)^{+}
$$

almost surely. Assume now that there are two optimal solutions $(\hat{\xi}, \hat{Z})$ and $(\tilde{\xi}, \tilde{Z})$ of the above linear programming problem. Due to the linearity of (4.21) it follows that any convex linear combination

$$
(\lambda \hat{\xi}+(1-\lambda) \tilde{\xi}, \lambda \hat{Z}+(1-\lambda) \tilde{Z})
$$

is also a solution to (4.21) for all $\lambda \in[0,1]$. In view of (4.22), we conclude that for each $\lambda \in[0,1]$

$$
\begin{aligned}
& \lambda(\Gamma+\Pi(\hat{\xi}))^{+}+(1-\lambda)(\Gamma+\Pi(\tilde{\xi}))^{+} \\
= & (\lambda(\Gamma+\Pi(\hat{\xi}))+(1-\lambda)(\Gamma+\Pi(\tilde{\xi})))^{+}
\end{aligned}
$$

holds almost surely. Since the above assertion is obviously violated on

$$
\{\Gamma+\Pi(\hat{\xi})<0<\Gamma+\Pi(\tilde{\xi})\} \cup\{\Gamma+\Pi(\hat{\xi})>0>\Gamma+\Pi(\tilde{\xi})\}
$$


this union must have a probability 0, which together with Assumption 2 yields (4.20). (iii) Assume on the contrary that there exists an equilibrium price process $S^{*}$ with

$$
S_{t}^{* k}(\omega)<\bar{S}_{t}^{k}(\omega) \text { for all } \omega \in B
$$

for some $t \in\{0,1, \ldots, T-1\}, B \in \mathcal{F}_{t}, P(B)>0$ and $k \in K$. Let $\xi^{*}$ be the corresponding equilibrium strategies. Since equilibrium allowance price $\bar{A}$ is unique it follows from (4.19) that

$$
\left\{S_{t}^{* k}-C_{t}^{i, j, k}-e^{i, j, k} \bar{A}_{t}<0\right\} \subseteq\left\{\xi_{t}^{* i, j, k}=0\right\}
$$

up to sets of probability zero. Consequently we obtain

$$
\begin{aligned}
\sum_{i \in I} \sum_{j \in J^{i, k}} \xi_{t}^{* i, j, k} & =\sum_{i \in I} \sum_{j \in J^{i, k}} \xi_{t}^{* i, j, k} 1_{\left\{S_{t}^{* k} \geq C_{t}^{i, j, k}+e^{i, j, k} \bar{A}_{t}\right\}} \\
& \leq \sum_{i \in I} \sum_{j \in J^{i, k}} \kappa^{i, j, k} 1_{\left\{S_{t}^{* k} \geq C_{t}^{i, j, k}+e^{i, j, k} \bar{A}_{t}\right\}}
\end{aligned}
$$

almost surely. Moreover it follows from (4.19) and (4.18) that

$$
\begin{aligned}
& \sum_{i \in I} \sum_{j \in J^{i, k}} \kappa^{i, j, k} 1_{\left\{\bar{S}_{t}>C_{t}^{i, j, k}+e^{i, j, k} \bar{A}_{t}\right\}} \\
= & \sum_{i \in I} \sum_{j \in J^{i, k}} \bar{\xi}_{t}^{i, j, k}-\sum_{i \in I} \sum_{j \in J^{i, k}} \bar{\xi}_{t}^{i, j, k} 1_{\left\{\bar{S}_{t}^{k}=C_{t}^{i, j, k}+e^{i, j, k} \bar{A}_{t}\right\}} \\
< & \sum_{i \in I} \sum_{j \in J^{i, k}} \bar{\xi}_{t}^{i, j, k}
\end{aligned}
$$

holds almost surely. In the last equality we used

$$
\sum_{i \in I} \sum_{j \in J^{i, k}} \bar{\xi}_{t}^{i, j, k} 1_{\left\{\bar{S}_{t}^{k}=C_{t}^{i, j, k}+e^{i, j, k} \bar{A}_{t}\right\}}(\omega)>0 \text { for all } \omega \in \Omega
$$

which follows from the definition of $\bar{S}$. Further due to (4.24) it holds that

$$
\begin{aligned}
& \sum_{i \in I} \sum_{j \in J^{i, k}} \kappa^{i, j, k} 1_{\left\{S_{t}^{* k} \geq C_{t}^{i, j, k}+e^{i, j, k} \bar{A}_{t}\right\}}(\omega) \\
& \leq \sum_{i \in I} \sum_{j \in J^{i, k}} \kappa^{i, j, k} 1_{\left\{\bar{S}_{t}^{k}>C_{t}^{i, j, k}+e^{i, j, k} \bar{A}_{t}\right\}}(\omega) \text { for all } \omega \in B .
\end{aligned}
$$

From (4.25), (4.26) and (4.27) we conclude that there exists a $C \subseteq B$ with

$$
\sum_{i \in I} \sum_{j \in J^{i, k}} \xi_{t}^{* i, j, k}(\omega)<\sum_{i \in I} \sum_{j \in J^{i, k}} \bar{\xi}_{t}^{i, j, k}(\omega)=D_{t}(\omega)
$$

for all $\omega \in C$, which implies that $S^{*}$ is no equilibrium product price. $\square$

REMARK 3. On the basis of what is known for merit-order equilibria with discontinuous cost functions, we do not expect uniqueness of the price process $S^{* k}$.

REMARK 4. In the introduction, we referred to social costs as the costs of regulation, i.e. the pollution reduction costs. We now give a formal definition of what we mean by social costs. For each regulatory allocation $\left(\left(\Lambda_{t}^{i}\right)_{t=0}^{T-1}\right)_{i \in I}$, and for any choice 
of an equilibrium production schedule $\xi^{*} \in \mathcal{U}$, we define the social costs $S C$ as the random variable given by the difference between the production costs $G\left(\xi^{*}\right)$ under this production schedule and the production costs incurred in the same random scenarios had we used the BAU equilibrium production schedule. In other words, the social costs are given by the random variable:

$$
S C=C\left(\xi^{*}\right)-C\left(\xi_{B A U}^{*}\right) .
$$

Notice also that as defined, the social costs do not depend upon the trading strategies of the individual firms in the emissions market.

REMARK 5. The results of this section were derived under the assumption that the emission coefficients $e^{i, j, k}$ were constant. However, by mere inspections of the proofs, the reader will easily convince herself that all the results remain true if these emission coefficients are instead adapted stochastic processes in $\mathcal{L}_{T-1}^{1}(\mathbb{R})$.

5. Prices and Windfall Profits in the Standard Scheme. The previous sections were dedicated to the introduction and the mathematical analysis of what we called the standard emission trading scheme. This cap-and-trade scheme was chosen because it is representative of the EU-ETS implementation.

In this section, we focus on an economy where one single good is produced. We choose the example of electricity because the power sector is worldwide one of the most important sources of green house gases. We study the impact of regulation on spot prices and producers' profits. In order to provide insight on the effects of capand-trade legislations, we performed numerical simulations of equilibrium prices and optimal production schedules by solving the global optimization problem (4.3) using data from the Texas electricity market. Specifics about the numerical implementation are given in the Appendix at the end of the paper. We shall report numerical findings from this case study throughout the remainder of the paper.

5.1. A Model for Electricity and Carbon Trading in Texas . To perform numerical simulations, we chose to focus on the electricity sector in Texas. Texas has an installed capacity of $81855 \mathrm{MW}$, mainly split into gas-fired (51489 MW), coal-fired (23321 MW), and nuclear (9019 MW) power plants. These figures are based on the installed capacity in 2007, including also additional nuclear and coal fired power plants that are planned to come online for the next 7 years. Including upcoming capacity slightly changes the production stack and leads to more interesting results than using the actual 2007 installed capacity. Nuclear technology has close to zero emissions, and it is always running in base-load. The source of emission reduction thus essentially comes from fuel switching between gas and coal.

So for all practical purposes, our model for Texas can be assume to involve one good, electricity, produced from two different technologies, gas and coal. Stochastic costs of production are equal to $C_{t}^{i, j, k}=H^{j} P_{t}^{j}$, where $j \in\{g, c\}, H^{j}$ is the heat rate of technology $j$ and $P_{t}^{j}$ is the corresponding fuel price. $D_{t}$ stands for the electricity demand from which nuclear capacity has already been subtracted. We set the emission rates to 0.42 ton/MWh for gas technology (CCGT-like) and 0.95 ton/MWh for coal technology respectively. These average emission rates have been chosen to give a faithful representation of Texas' park of power plants.

The global optimization problem reads:

$$
\inf _{\xi \in \mathcal{U}} \mathbb{E}\left[\sum_{t=0}^{T-1}\left(C_{t}^{g} \xi_{t}^{g}+C_{t}^{c} \xi_{t}^{c}\right)+\pi\left(\sum_{t=0}^{T-1}\left(e^{g} \xi_{t}^{g}+e^{c} \xi_{t}^{c}\right)-\Gamma_{0}\right)^{+}\right]
$$


under the constraint: $\xi_{t}^{g}+\xi_{t}^{c}=D_{t}$ for every time $t$. In the particular case of two technologies, we can proceed to the change of variable $\left(\xi_{t}^{g}, \xi_{t}^{c}\right) \longmapsto\left(\mathcal{E}_{t}, \mathcal{C}_{t}\right)$, where

$$
\mathcal{E}_{t}=e^{c} \xi_{t}^{c}+e^{g} \xi_{t}^{g} \quad \text { and } \quad \mathcal{C}_{t}=C_{t}^{c} \xi_{t}^{c}+C_{t}^{g} \xi_{t}^{g}
$$

are respectively the total emission and the cost of production for the period $[t, t+$ 1). Using the constraint that the demand has to be met, we obtain an equivalent formulation in terms of an emission abatement problem:

$$
\min _{\underline{\mathcal{E}} \leq \mathcal{E} \leq \overline{\mathcal{E}}} \mathbb{E}\left[\sum_{t=0}^{T-1}\left(D_{t}\left(e^{c} F_{t}+C_{t}^{c}\right)-F_{t} \mathcal{E}_{t}\right)+\pi\left(\sum_{t=0}^{T-1} \mathcal{E}_{t}-\Gamma_{0}\right)^{+}\right]
$$

where:

$$
\begin{aligned}
& \underline{\mathcal{E}}_{t}=e^{g} \min \left(D_{t}, \kappa^{g}\right)+e^{c}\left(D_{t}-\kappa^{g}\right)^{+} \\
& \overline{\mathcal{E}}_{t}=e^{c} \min \left(D_{t}, \kappa^{c}\right)+e^{g}\left(D_{t}-\kappa^{c}\right)^{+}
\end{aligned}
$$

are respectively the maximal and emissions possible at time $t$, and

$$
F_{t}:=\frac{C_{t}^{g}-C_{t}^{c}}{e^{c}-e^{g}}
$$

is the fuel spread per ton of $\mathrm{CO}_{2}$ (or abatement cost). The fuel spread $F$ represents the marginal switching cost necessary to decrease emissions by 1 unit. We observe that the above formulation (5.1) only involves 2 exogenous stochastic processes: $D$ and $F$. Finally, we set the aggregated uncontrolled emissions $\sum_{i \in I} \Delta^{i}$ infinitesimally small to stay in the realm of the assumptions of Theorem 4.6, and solve the global optimization problem by stochastic dynamic programming on a 2-dimensional trinomial tree. Details are given in the appendix at the end of the paper.

5.2. Electricity Prices Under the Standard Scheme. In this subsection, we discuss the impact of the regulation on electricity prices. We already emphasized that uniqueness of equilibrium electricity prices was not granted. However, we identified the minimal price among all the possible equilibrium prices in Equation (4.15). In what follows, we focus on this price.

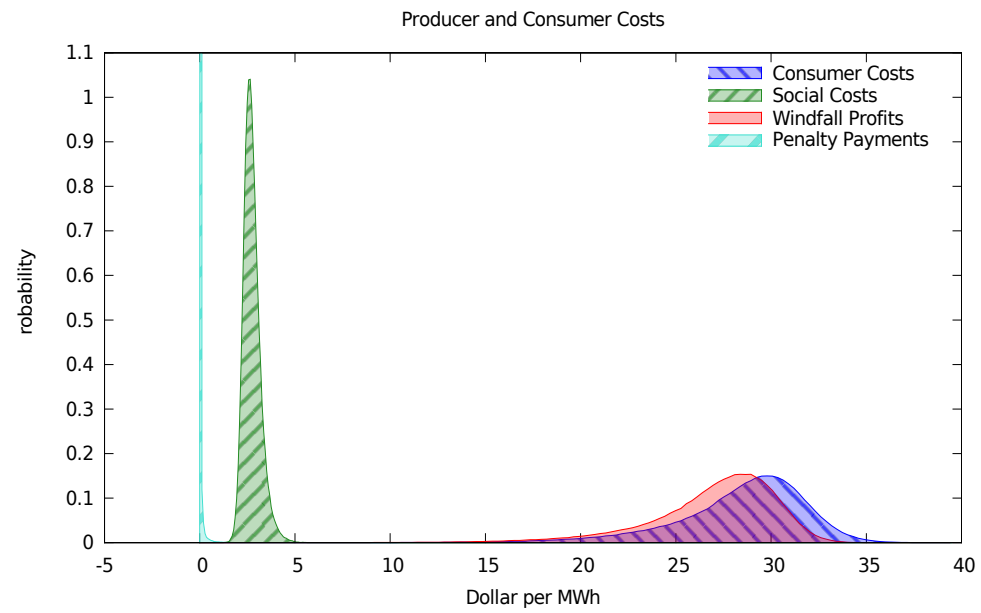

FiguRE 5.1. Histograms of the consumer costs, social costs, windfall profits and penalty payments under a standard trading scheme scenario. 
Equation (4.15) shows two sources of change in the spot price compared to business as usual. First, the marginal technology may be different: this induces a variation in marginal cost. This variation is likely to be positive but a negative variation is possible. Suppose for example that in a BAU scenario, coal is started first but that demand is high enough so that gas is the marginal technology. Suppose that in the presence of the trading scheme, allowance price is high enough to induce a fuel switch, so that gas is started first. Assume also that demand is high so that coal is the marginal technology. In this case, the variation in marginal cost can be negative. The second source of variation is the price of pollution $e^{i, j, k} A_{t}^{*}$ for the marginal technology. The producers pass through the cost of expected penalties to end-consumers. This second contribution is always positive and is such that the spot price under the trading scheme is always greater than the spot price in BAU.

A possible interpretation of formula (4.15) is that the allowance price enters the electricity price as the price of an additional commodity that is used for power generation besides fuels. Producing the last infinitesimal unit of electricity at time $t$ induces not only costs due to extra fuel consumption, but also increases the emissions by $e^{i, j, k}$ and hence also the expected penalty at time $T$ by $e^{i, j, k} A_{t}^{*}$. Consequently these costs have to be covered by the end-consumers, for the marginal production of product $k$ to be profitable. Since this amount is passed on to the endconsumer in each timestep the consumer cost $\sum_{t=0}^{T-1}\left(S_{t}^{*}-S_{t}^{B A U}\right) D_{t}$ are much bigger than the penalty that is actually paid. As we will see in the following the consumer costs exceed also by far the social cost of the scheme.

Figure 5.1 quantifies both the penalty payments and the consumer cost and compares them to social costs and windfall profits (as defined in the next section) under a standard trading scheme for the Texas electricity sector. The penalty and initial allocation for this example are $\pi=100 \$$ and $\theta_{0}=1.826 \times 10^{8}$ allowances respectively. This allocation corresponds to a reduction target of $10 \%$, i.e. $1.827 \times 10^{8} \mathrm{t}$ Carbon, to be reached with $95 \%$ probability.

The results depicted in Figure 5.1 illustrate the major critic articulated by some of the opponents of the cap-and-trade systems. We observe that end consumer costs, are approximately more than 10 times higher than social costs due to the trading scheme. Hence the consumers' burden exceeds by far the the overall reduction costs, which gives rise for significant extra profits for the producers.

5.3. Windfall Profits and Penalty Under the Standard Scheme. As explained above, the pricing mechanism of the standard emissions trading scheme induces a significant wealth transfer from consumers to producers.

Another way of understanding the extra profits made by the producers is to consider the windfall profits defined as follows. In the general framework of a standard cap-and-trade system with multiple goods introduced earlier, if $\xi^{*}$ is an optimal production strategy associated with the equilibrium $\left(A^{*}, S^{*}\right)$, we define the target price $\hat{S}_{t}^{k}$ of good $k$ as:

$$
\hat{S}_{t}^{k}:=\max _{i \in I, j \in J^{i, k}} C_{t}^{i, j, k} \mathbf{1}_{\left\{\xi_{t}^{* i, j, k}>0\right\}} .
$$

This price is the marginal cost under the optimal production schedule without taking into account the cost of pollution. We then define the windfall profits of firm $i$ as:

$$
\sum_{t=0}^{T-1} \sum_{(j, k) \in M_{i}}\left(S_{t}^{* k}-\hat{S}_{t}^{k}\right) \xi_{t}^{* i, j, k},
$$


and the overall windfall profits as

$$
W P=\sum_{t=0}^{T-1} \sum_{k \in K}\left(S_{t}^{* k}-\hat{S}_{t}^{k}\right) D_{t}^{k}
$$

These windfall profits measure the profits for the production of goods in excess over what the profits would have been, had the same dispatching schedule been used, and the target prices (e.g. the marginal fuel costs) be charged to the end consumers without the cost of pollution.

REMARK 6. Another reasonable definition of the windfall profits of firm $i$ would be

$$
\sum_{t=0}^{T-1} \sum_{(j, k) \in M_{i}}\left(S_{t}^{* k}-\hat{S}_{t}^{k}\right) \xi_{t}^{* i, j, k}-\pi\left(\Gamma^{i}-\Pi^{i}\left(\xi^{* i}\right)\right)^{+}
$$

meaning that the penalty payments due to the scheme are withdrawn from the extra profits. Since producers decide upon their production strategy and therewith the risk to pay the penalty, we take the point of view that they should pay the penalty and not the endconsumer. However as can be seen in Figure 5.1 the penalty payments vanish in comparison to the windfall profits as defined in (5.4). Hence in practical applications, both definitions should give similar results.

Figure 5.1 shows the distribution of windfall profits as computed in the example of the Texas electricity market chosen for illustration purposes. We observe that the windfall profits are in average almost 10 times higher than actual abatement costs. Furthermore it also shows that the costs of expected future penalty passed to the customers are much higher (4637 times) than the penalty actually paid. This is consistent with the deterministic example presented in the introduction.

5.4. Incentives for Cleaner Technologies. Using (4.17) we see that the expected profits and losses of firm $i \in I$ in an equilibrium $\left(A^{*}, S^{*}\right)$ with associated production schedules $\xi^{*}$ are given by

$$
\begin{aligned}
\mathbb{E}\left[L^{A^{*}, S^{*}, i}\left(\theta^{* i}, \xi^{* i}\right)\right]=\mathbb{E} & {\left[\left(-\Delta^{i}+\sum_{t=0}^{T-1} \Lambda_{t}^{i}\right) A_{T}^{*}\right] } \\
+ & \mathbb{E}\left[\sum_{t=0}^{T-1} \sum_{(j, k) \in M_{i}}\left(S_{t}^{* k}-C_{t}^{i, j, k}-e^{i, j, k} A_{t}^{*}\right) \xi_{t}^{* i, j, k}\right]
\end{aligned}
$$

As will be shown in Proposition 7.1, both the equilibrium price processes $\left(A^{*}, S^{*}\right)$ and the production strategies $\xi^{*}$ are preserved under a change of the regulatory allocation from $\left(\left(\Lambda_{t}^{i}\right)_{t=0}^{T-1}\right)_{i \in I}$ to $\left(\left(\tilde{\Lambda}_{0}^{i}\right)_{t=0}^{T-1}\right)_{i \in I}$ as long as

$$
\sum_{i \in I} \sum_{t=0}^{T-1} \Lambda_{t}^{i}=\sum_{i \in I} \sum_{t=0}^{T-1} \tilde{\Lambda}_{t}^{i}
$$

holds almost surely. However, such an adjustment of the allocation changes the expected profits and losses of producer $i \in I$ by the amount:

$$
\mathbb{E}\left[\left(\tilde{\Lambda}_{0}^{i}-\Lambda_{0}^{i}\right) A_{T}^{*}\right]
$$


Obviously this gives a relative (relative to $\Lambda_{0}^{i}$ ) expected money transfer of

$$
\mathbb{E}\left[\sum_{i \in I}\left(\tilde{\Lambda}_{0}^{i}-\Lambda_{0}^{i}\right)^{+} A_{T}^{*}\right]
$$

from producers with $\tilde{\Lambda}_{0}^{i}-\Lambda_{0}^{i}<0$ to producers with $\tilde{\Lambda}_{0}^{i}-\Lambda_{0}^{i}>0$. If the initial allocation is given depending on the type of production plant it is possible to utilize this mechanism to increase or decrease the incomes of clean and dirty plants respectively, i.e. the initial allocation can be used to adjust the incentives to build cleaner plants. Depending on the specific market this will often be the main incentive to build clean plants.

This mechanism is one of the main features of cap and trade schemes and will in general fail if auctioning is used to abolish windfall profits. First notice that even a $100 \%$ auction can not always reduce windfall profits to zero. This becomes obvious in a market with a lot of nuclear power plants where coal is marginal the whole time. In such a market the producers of nuclear power make huge windfall profits but since their emissions are zero they do not need any allowances. Hence the auction can only cover the windfall profits due to the coal fired plants. Therefore using auctioning to cut windfall profits a huge amount if not all allowances of the initial allocation should be auctioned. However in such a case, the regulator looses the instrument to control above incentives. Therefore in the next section we propose alternative cap and trade schemes that not only reduce windfall profits to zero in average, but also provide a considerable amount of allowances that can be used to adjust incentives to build cleaner plants.

6. Alternative Designs of Emission Trading Schemes. The main objectives of emission trading schemes are both to force the market to reach a certain reduction target, and at the same time, to give incentives to develop and build cleaner production facilities. In view of the shortcomings of the standard cap-and-trade scheme demonstrated in the last section, we propose alternative designs which fulfil both objectives at low social costs, low windfall profits and hence low costs transfered to the consumer.

This is possible because the mathematical theory developed in the previous sections allows us to study emissions reduction policies that are different from the standard EU-ETS scheme.

In the first Subsection 6.1 below, we introduce a general (and fairly complex) cap-and-trade scheme including taxes and subsidies. We argue that the theoretical results derived earlier in the paper for standard schemes, still hold in this more general situation. The remaining of the section is devoted to the identification and the calibration of two of the simplest particular cases of interest. A relative scheme is introduced in Subsection 6.2 and a tax scheme is introduced in Subsection 6.3. The final Subsection 6.4 provides comparative statics highlighting the differences between these schemes on the case study of the Texas electricity market.

6.1. General Market Designs for Emission Trading Schemes. We describe the new regulator policies by first generalizing the allocation procedure. Beyond the static allocation $\Lambda_{t}^{i}$ for firm $i$ at time $t$, the regulator is now allowed to distribute credits dynamically and proportionally to production. To be more specific, at each time $0 \leq t<T$, firm $i$ is provided with an allocation

$$
\Lambda_{t}^{i}=X_{t}^{i}+\sum_{(j, k) \in M(i)} Y_{t}^{i, j, k} \xi_{t}^{i, j, k},
$$


where $X^{i}$ and $Y^{i, j, k}$ are adapted processes in $\mathcal{L}_{T-1}^{1}(\mathbb{R})$. For the sake of generality we let $Y_{t}^{i, j, k}$ depend upon $j$. However in this case the opportunity to relate the number of allowances to real emissions is lost.

In addition, the regulator can also tax or subsidize the various firms by means of financial incentives or disincentives similar to the credit endowments described above. In this case, the firms' profits are lowered at time $t$ by an amount

$$
T S^{i}=V_{t}^{i}+\sum_{(j, k) \in M(i)} Z_{t}^{i, j, k} \xi_{t}^{i, j, k}
$$

where $V^{i}$ and $Z^{i, j, k}$ are as before, adapted processes in $\mathcal{L}_{T-1}^{1}(\mathbb{R})$. Remark that $V^{i}$ and $Z^{i, j, k}$ stand for a tax when positive and a subsidy when negative. Examples of positive $Z^{i, j, k}$ include fuel and $\mathrm{CO}_{2}$ taxes. The combination of $V^{i}$ and $Z^{i, j, k}$ allows for the introduction of alternative regulation such as a system of reward/penalty with respect to a given production (or equivalently emission) target $\underline{\xi}^{i, j, k}$. By charging the quantity

$$
\sum_{t=0}^{T-1} \sum_{(j, k) \in M_{i}} Z_{t}^{i, j, k}\left(\xi_{t}^{i, j, k}-\underline{\xi}_{t}^{i, j, k}\right)
$$

corresponding to $V_{t}^{i}=-\sum_{(j, k) \in M_{i}} Z_{t}^{i, j, k} \underline{\xi}_{t}^{i, j, k}$, the regulator can provide incentives for firm $i$ to stay close to a given production or emission strategy.

Under such a generalized cap-and-trade scheme, the terminal wealth (or profits and losses) of firm $i \in I$ reads:

$$
\begin{aligned}
L^{A, S, i}\left(\theta^{i}, \xi^{i}\right) & :=-\sum_{t=0}^{T-1} V_{t}^{i}+\sum_{t=0}^{T-1} \sum_{(j, k) \in M_{i}}\left(S_{t}^{k}-C_{t}^{i, j, k}-Z_{t}^{i, j, k}\right) \xi_{t}^{i, j, k} \\
& +\sum_{t=0}^{T-1} \theta_{t}^{i}\left(A_{t+1}-A_{t}\right)-\theta_{T}^{i} A_{T} \\
& -\pi\left(\Delta^{i}+\Pi^{i}\left(\xi^{i}\right)-\sum_{t=0}^{T-1}\left(X_{t}^{i}+\sum_{(j, k) \in M(i)} Y_{t}^{i, j, k} \xi_{t}^{i, j, k}\right)-\theta_{T}^{i}\right)^{+} .
\end{aligned}
$$

Despite the obviously greater generality of the present framework, the proofs of the results of Theorem 4.6 are sufficient to cover the analysis of this broader class of trading schemes:

Proposition 6.1. If we set

$$
\hat{\Gamma}^{i}:=\Delta^{i}-\sum_{t=0}^{T-1} X_{t}^{i}, \quad \hat{e}_{t}^{i, j, k}:=e^{i, j, k}-Y_{t}^{i, j, k}, \quad \text { and } \quad \hat{C}_{t}^{i, j, k}:=C_{t}^{i, j, k}+Z_{t}^{i, j, k}
$$

for a set of adjusted parameters, then the results of Theorem 4.6 hold true in the case of the the generalized cap-and-trade scheme of this subsection provided we replace the parameters of Theorem 4.6 by the adjusted parameters so-defined.

Proof. The proof of this proposition follows a straightforward adaptation of the arguments used in the previous sections and Remark 5 about stochastic emission factors. 
The present formulation gives a general framework for the analysis of a broader class of cap-and-trade schemes. We mostly focus on two important particular cases: 1) the case where $Z_{t}^{i, j, k} \geq 0$ varies with $i$ and $j$ which represents a fuel or emission tax scheme, and 2) the case where $Z_{t}^{i, j, k} \leq 0$ only depends on $k$, which corresponds to a subsidy for the production of good $k$.

For an equilibrium $\left(A^{*}, S^{*}\right)$ of the generalized scheme with associated strategies $\left(\theta^{*}, \xi^{*}\right)$ it is straightforward to extend the definition of windfall profits of firm $i$ as:

$$
G W P^{i}=\sum_{t=0}^{T-1} \sum_{(j, k) \in M_{i}}\left(S_{t}^{* k}-\hat{S}_{t}^{k}\right) \xi_{t}^{* i, j, k}-\sum_{t=0}^{T-1}\left(V_{t}^{i}+\sum_{(j, k) \in M_{i}} Z_{t}^{i, j, k} \xi_{t}^{* i, j, k}\right),
$$

the overall windfall profits being then defined as

$$
G W P=\sum_{t=0}^{T-1} \sum_{k \in K}\left(S_{t}^{* k}-\hat{S}_{t}^{k}\right) D_{t}^{k}-\sum_{t=0}^{T-1}\left(V_{t}^{i}+D_{t}^{k} \sum_{i \in I, j \in J^{i, k}} Z_{t}^{i, j, k}\right) .
$$

The above discussion suggests that windfall profits could be reduced with a relative allocation rule constant over time. This motivates the following analysis.

6.2. Cap-and-Trade Schemes with Relative Allowance Allocation. A positive relative allocation for some product $Y_{t}^{i, j, k}=y^{k}<0$ for some $k \in K$ all $i \in I$ and those $j \in J^{i, k}$ can be seen as a subsidy for good $k$ that is given in the form of allowances rather than in cash. When producing one unit of good $k$, the marginal penalty increases only by $\left(e^{i, j, k}-y^{k}\right) A_{t}^{*}$ rather than by $e^{i, j, k} A_{t}^{*}$ as in a standard scheme. Thus the net marginal overall production costs of the firms are lower when compared to the standard scheme. This should result in a decrease of the price of good $k$. In the present subsection, we study the simplest generalized cap-and-trade scheme taking advantage of this mechanism. It applies this mechanism only for production means $J_{\text {marg }}^{i, k} \subseteq J^{i, k}$ which can be marginal (e.g. in the case of the electricity markets, this excludes nuclear plants) and is obtained by setting:

$$
\begin{aligned}
Y_{t}^{i, j, k} & =y^{k} 1_{\left\{j \in J_{\text {marg }}^{i, k}\right\}} \in \mathbb{R} \quad \text { for all } t=0, \ldots, T-1 \\
X_{0}^{i} & =x^{i} \in \mathbb{R} \quad, \quad X_{t}^{i}=0 \quad \text { for all } t=1, \ldots, T-1 \\
V_{t}^{i} & =0, \quad Z_{t}^{i, j, k}=0 \quad \text { for all } t=0, \ldots, T-1
\end{aligned}
$$

for all $(i, j, k) \in M$.

In what follows, not only do we discuss this relative cap-and-trade scheme, but we also gain new insight into the standard cap-and-trade scheme by treating it as a relative cap-and-trade scheme with $y^{k}=0$ for all $k \in K$.

But for any comparison of different cap-and-trade schemes to be meaningful, we need to calibrate their respective parameters to common characteristics. We proceed to the discussion of such a calibration.

6.2.1. Calibration of the Parameters. The relative scheme has three regulatory parameters. Using the notation of this section, they are: 1) the penalty $\pi, 2$ ) the relative allocation coefficients $\left.\left(y^{k}\right)_{k \in K}, 3\right)$ the total initial allocations $x=\sum_{i \in I} x^{i}$ given to the firms $i \in I$. In this subsection we show, using again the example of the Texas electricity market, how one should choose these parameters in order to guarantee an emissions reduction target with given probability while keeping the expected windfall profits near zero and controlling the social costs to keep them as low 

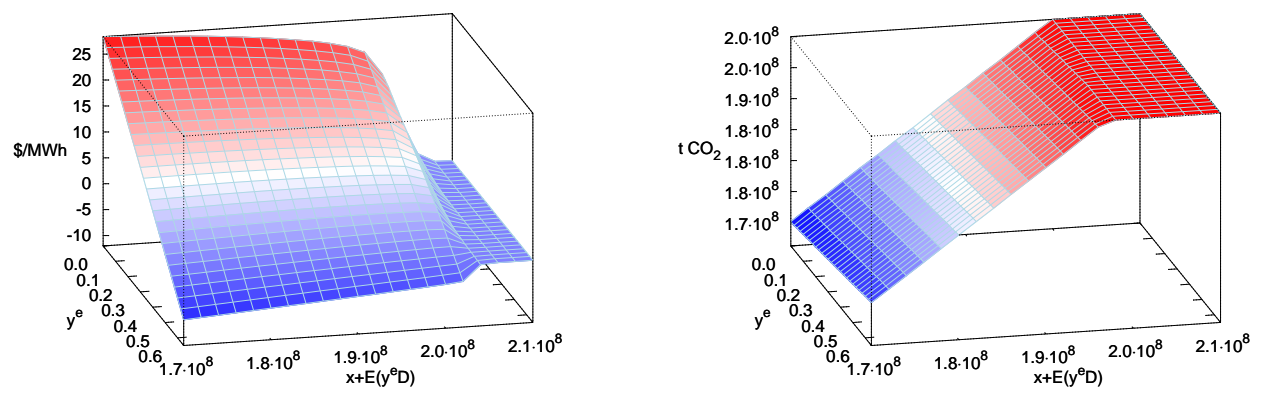

FIGURE 6.1. Windfall profits (left) and $95 \%$ percentile of total emissions (right) as functions of the relative allocation parameter and the expected allocation. Here $D=\sum_{t=0}^{T-1} D_{t}^{e}$ denotes the total electricity demand over one compliance period.

as possible. In the particular simulation used to illustrate the strategy, we choose an emissions reduction target of $1.827 \times 10^{8}$ to be reached with probability $95 \%$.

To gain a first insight into the numerics, we fix the penalty $\pi$ at $100 \$$. The left pane of Figure 6.1 gives the expected windfall profits while the right pane gives the $95 \%$ percentile of the total emissions for different values of the relative allocation coefficient $\left(y^{e}\right)$ and the expected total allocation. It appears that the expected allocation controls the amount by which carbon emissions are reduced, while the relative allocation coefficient $y^{e}$ controls the windfall profits. Designing a cap-and-trade scheme with zero windfall profits and pre-decided emissions target levels can be done by choosing the parameters of our relative scheme at the intersection of the zero windfall profit level set with the $1.827 \times 10^{8}$ emission percentile level set. This procedure is depicted in Figure 6.2. We find $y^{e}=0.54$ and $\sum_{i \in I} x^{i}=5.4 \times 10^{7}$. 

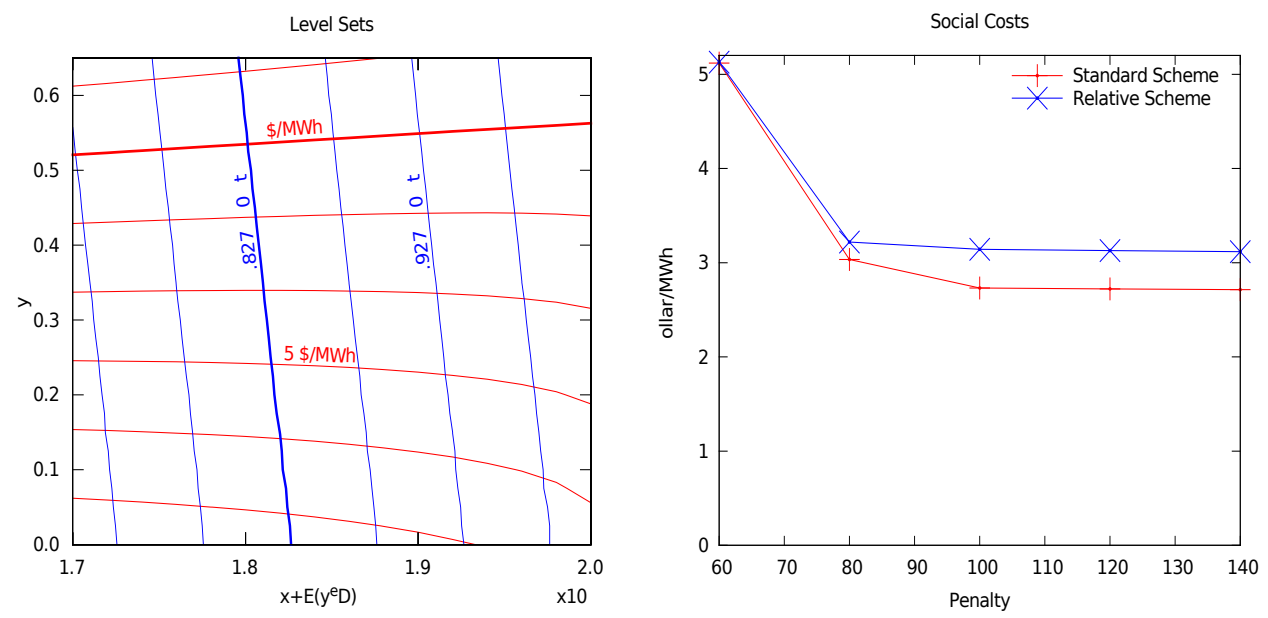

Figure 6.2. The left pane shows the level sets of the two plots of Figure 6.1. The blue and the red lines indicate level sets of the windfall profits and the $95 \%$ quantile of emissions respectively. The right pane gives the plots of the overall production costs for electricity for one year as function of the penalty level for both the absolute and relative schemes. The free regulatory parameters are chosen to guarantee the desired emissions percentile, and in the case of the relative scheme, such that the windfall profits are zero.

Since for the standard cap-and-trade scheme the parameter $y^{e}=0$ is fixed, we have one less regulatory parameter. Thus controlling the emissions level by the initial allocation, we are not able to control the windfall profits. Hence the desired parameter values are obtained at the intersection of the $1.827 \times 10^{8}$ emission percentile level set with $y^{e}=0$. Giving the initial allocation $\sum_{i \in I} x^{i}=1.826 \times 10^{8}$.

Repeating the above procedure for different penalties levels gives regulatory settings with different production costs for the relative and the standard scheme in Figure 6.2. Obviously for both schemes the social costs are reduced by increasing penalty. As shown in the right pane of Figure 6.2, this decrease in social costs is significant until the penalty reaches the level $\pi=100 \$$. after that, the social costs stay nearly the same, becoming independent of $\pi$ for larger values of $\pi$. Hence, we conclude that in this setting a penalty of $100 \$$ is a reasonable choice for both the relative and the standard scheme.

6.3. Emission Taxes. A static tax scheme is a regulation that penalizes the emission of each ton of carbon by a fixed amount, say $z>0$. Formally, it can be viewed as a generalized scheme for which

$$
\begin{aligned}
& Z_{t}^{i, j, k}=e^{i, j, k} z \quad, \quad V_{t}^{i}=0 \\
& Y_{t}^{k}=0 \quad, \quad X_{t}^{i}=0
\end{aligned}
$$

for all $(i, j, k) \in M$ and $t=0, \ldots, T-1$. Using the results of Proposition 6.1, we see that in such a tax scheme the prices of goods follow a merit order pricing rule with effective production costs given by $C_{t}^{i, j, k}+e^{i, j, k} z$ for all $(i, j, k) \in M$ and $t=0, \ldots, T-1$. The earnings under a tax scheme are based on the spread of these effective production costs. Since this spread does not depend solely on the original fuel spread, it is in general not clear what windfall profits will be. It is not even clear if they are negative or positive. To gain some insight on this issue consider a tax of 
$z=60 \$$ (which is realistic for a $10 \%$ reduction target as will be seen below), and assume that at some point in time, the marginal production costs of coal and gas are the same while all plants have to run to satisfy the demand. In this case the spread in effective production cost is $\left(e_{c}-e_{g}\right) z=31.8 \$$ and will be earned for each MWh that is produced with gas. However, in the case of BAU, the earnings are zero. Hence the windfall profits are $31.8 \$$ per MWh of electricity produced with gas.

In tax schemes the only regulatory control parameter $z$ should be adjusted in order to guarantee a specified reduction target. Thus the windfall profits are automatically given by the reduction target and can not be adjusted.

6.4. Comparison of the Various Abatement Schemes. We now compare the characteristics of the standard and the relative cap-and-trade schemes with the regulatory parameters chosen in the previous subsection.

We first consider the windfall profits and the consumer costs. The results are given in Figure 6.3.
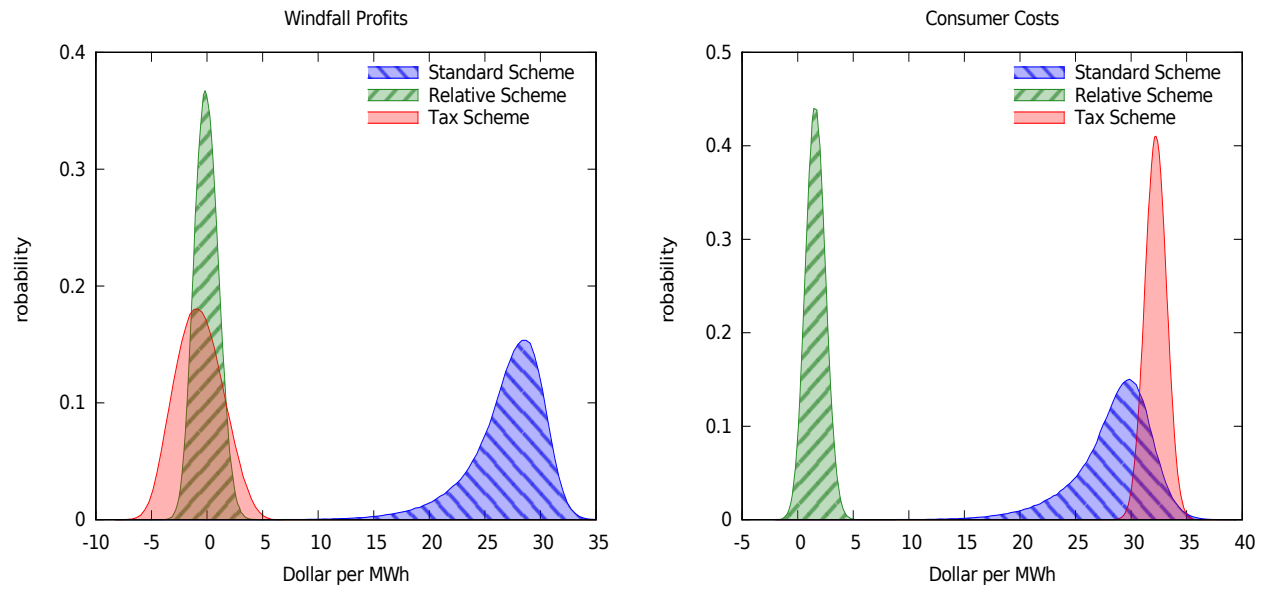

FIGURE 6.3. Histograms (computed from 500000 simulation scenarios) of the yearly distribution of windfall profits (left) and consumer costs (right) for the Standard Scheme, a Relative Scheme and a Tax Scheme.

As expected the relative scheme gives much lower consumer costs than the standard scheme. This is related to the fact that the windfall profits have a narrow distribution around zero in the case of the relative scheme, while the windfall profits of the standard scheme are 10 times higher than the social costs. When compared to the standard scheme, the only drawback of the relative scheme seems to be the slightly higher level of social costs which can be observed on the right pane of Figure 6.4. However since this cost increase corresponds to approximately $0.4 \$$ per $\mathrm{MWh}$ it is small in comparison to production costs and thus can be neglected in practice. Moreover those higher production costs are not just wasted money, they are paid for higher emission reduction in many scenarios as can be seen on the left pane of Figure 6.4. In particular the relative scheme takes advantage of cheap fuel switches when the standard scheme cannot reduce emissions anymore. Moreover relative scheme is less sensitive to weather, since in warm winters less allowances are allocated pushing the price up. This in turn is responsible for higher emission abatements and consequently 

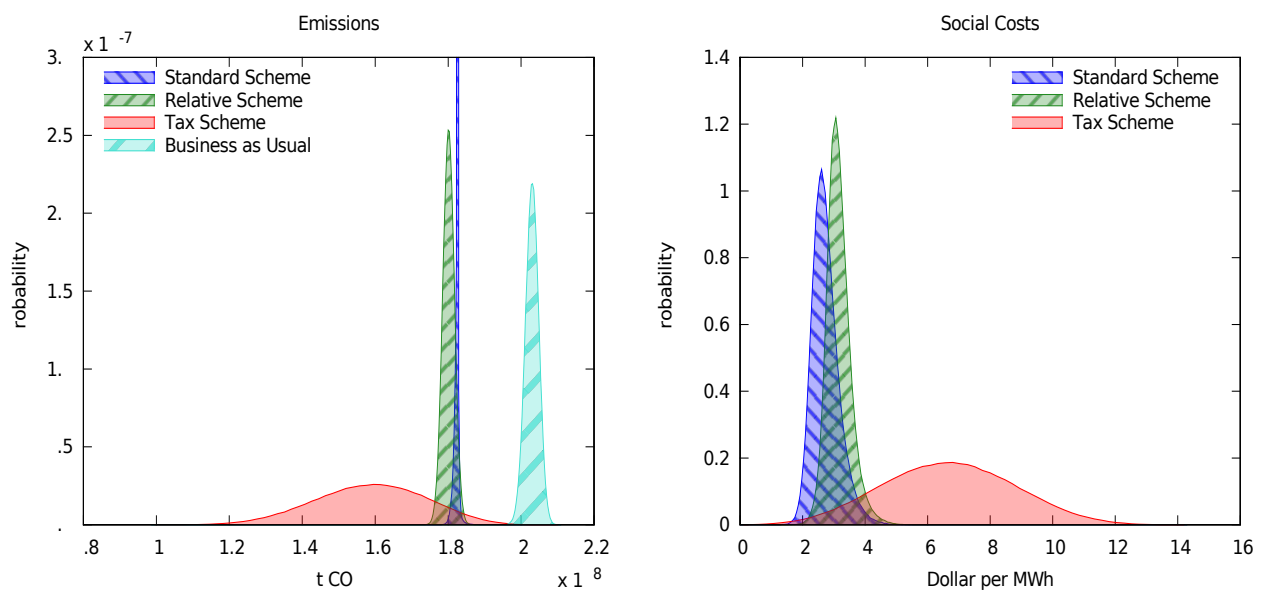

FIGURE 6.4. Yearly emissions from electricity production (left) for the Standard Scheme, the Relative Scheme, a Tax Scheme and BAU, and yearly abatement costs (right).

higher abatement costs.

In this example approximately $30 \%$ of the allowances are given as initial allocation, by allocating these to clean plants, further incentives can be set to build cleaner plants. This seems to be an important advantage of the relative scheme over other mechanisms such as auctioning and tax.

Next, we study the effect of an emission tax on the Texas electricity market. Figure 6.4 shows that a pure tax scheme that fulfills the above reduction target of $1.827 \times 10^{8} t_{\mathrm{CO}_{2}}$ with $95 \%$ probability, is on average, more than twice $(2.4 \times)$ as expensive as the standard cap and-trade-scheme. In other words, it has a poor emissions reduction performance. These extra costs are paid for extra emission reductions. However in contrast with the results in the case of the relative scheme, the average cost increase per reduced ton of carbon is considerable when we compare it to the case of the standard scheme. The reason is that a tax is not flexible enough to control emissions when abatement costs are stochastic. This results in an emission uncertainty that exceeds even the BAU uncertainty with several orders of magnitude. Notice moreover that it carries a significant risk to reduce nearly no emissions. In such a scenario the tax corrections for upcoming years will be extremely expensive. Needless to say a tax scheme induces a huge money transfer from consumers to the regulator, which as can be seen in Figure 6.4 is even bigger than the costs transferred to the consumer in a standard cap and trade scheme.

7. More Financial Incentives. One of the main arguments in favor of the relative schemes studied in the previous section is the fact that they reduce windfall profits. However, this reduction comes with slightly higher reduction costs than in the case of the absolute scheme. While this cost increase is negligible in practice, it is of great theoretical interest to understand how and why one can design schemes that give exactly zero windfall profits at exactly the same reduction costs as the standard cap-and-trade scheme. In order to do so, we need to identify the generalized schemes which are in a one-to-one correspondence with the production policies of the standard 
scheme. The latter are given by a subclass of generalized schemes for which $Z_{t}^{i, j, k}$ and $Y_{t}^{i, j, k}$ depend only on $k$. The terminal wealth of firm $i \in I$ under such a scheme reads:

$$
\begin{aligned}
L^{A, S, i}\left(\theta^{i}, \xi^{i}\right) & :=-\sum_{t=0}^{T-1} V_{t}^{i}+\sum_{t=0}^{T-1} \sum_{(j, k) \in M_{i}}\left(S_{t}^{k}-C_{t}^{i, j, k}-Z_{t}^{k}\right) \xi_{t}^{i, j, k} \\
& +\sum_{t=0}^{T-1} \theta_{t}^{i}\left(A_{t+1}-A_{t}\right)-\theta_{T}^{i} A_{T} \\
& -\pi\left(\Delta^{i}+\Pi^{i}\left(\xi^{i}\right)-\sum_{t=0}^{T-1}\left(X_{t}^{i}+\sum_{(j, k) \in M(i)} Y_{t}^{k} \xi_{t}^{i, j, k}\right)-\theta_{T}^{i}\right)^{+} .
\end{aligned}
$$

The results of this section will demonstrate the versatility and the flexibility of the generalized framework introduced in this paper. However, because of the level of complexity of their implementations, and despite the high degree of control they provide the regulator with, it is unlikely that the schemes identified here will be used by policy makers or regulators.

7.1. Equilibria Equivalence. Our first result exhibits a one-to-one correspondence between the equilibria of standard schemes and generalized schemes leading to profits and losses for firm $i$ of the type (7.1).

Proposition 7.1. If $\left(A^{*}, S^{*}\right)$ is an equilibrium with production strategies $\xi^{*}$ for a standard cap-and-trade scheme with adjusted uncontrolled emissions given by

$$
\Gamma^{i}=\Delta^{i}-\sum_{s=0}^{T-1}\left(X_{s}^{i}+\Xi_{s}^{i}\right) \quad \text { for all } i \in I,
$$

where $\Xi$ is a stochastic process in $\mathcal{L}_{T-1}^{1}(\mathbb{R})$ such that

$$
\sum_{t=0}^{T-1} \sum_{i \in I} \Xi_{t}^{i}=\sum_{t=0}^{T-1} \sum_{k \in K} Y_{t}^{k} D_{t}^{k},
$$

then the prices $\left(A^{*}, S^{\dagger}\right)$ where

$$
S_{t}^{\dagger k}=S_{t}^{* k}+Z_{t}^{k}-Y_{t}^{k} A_{t}^{*} \quad \text { for all } k \in K, t=0, \ldots, T-1
$$

define an equilibrium of the generalized cap-and-trade scheme with the same production strategies $\xi^{*}$. The converse statement also holds.

In particular if

$$
\sum_{t=0}^{T-1}\left(\sum_{i \in I} X_{t}^{i}+\sum_{k \in K} Y_{t}^{k} D_{t}^{k}\right)=\sum_{i \in I} \Lambda_{0}^{i}
$$

where $\Lambda_{0}^{i}$ is the allocation in standard scheme, there is a one-to-one correspondence between the generalized scheme and the standard scheme with initial allocation $\Lambda_{0}^{i}$.

In particular, the lowest equilibrium product price is given by (7.4) where $S^{*}$ is given by Theorem 4.6 for the standard scheme with adjusted uncontrolled emissions $\left(\hat{\Gamma}^{i}\right)_{i \in I}$. To prove Proposition 7.1, we shall need the following lemma. 
Lemma 7.2. Let $A$ be an integrable martingale, $\theta, \theta^{\prime} \in \mathcal{V}^{i}(A), \xi^{i} \in \mathcal{U}^{i}$, and $S, S^{\prime}$ be two integrable price processes, such that:

$$
\begin{aligned}
{\theta^{\prime}}^{i} & =\theta_{t}^{i} \quad \text { for all } t \leq T-1 \\
{\theta^{\prime}}_{T}^{i} & =\theta_{T}^{i}-\sum_{t=0}^{T-1}\left(\Xi_{t}^{i}-\sum_{(j, k) \in M(i)} Y_{t}^{k} \xi_{t}^{i, j, k}\right) \\
{S^{\prime}}^{k} & =S_{t}^{k}+Z_{t}^{k}-Y_{t}^{k} A_{t} \quad \text { for all } k \in K, t=0, \ldots, T-1
\end{aligned}
$$

where $Z^{k} \leq 0$ is a subsidy, then we have:

$$
\mathbb{E}\left[L^{A, S, i}\left(\theta^{i}, \xi^{i}\right)\right]=\mathbb{E}\left[H^{A, S^{\prime}, i}\left(\theta^{\prime i}, \xi^{i}\right)\right]+\mathbb{E}\left[\sum_{t=0}^{T-1}\left(V_{t}^{i}-\Xi_{t}^{i} A_{t}\right)\right] .
$$

Proof. The martingale property of $A$ yields:

$$
\begin{aligned}
& \mathbb{E}\left[\theta_{T}^{i} A_{T}+\pi\left(\Delta^{i}+\Pi^{i}\left(\xi^{i}\right)-\sum_{t=0}^{T-1}\left(X_{t}^{i}+\sum_{(j, k) \in M(i)} Y_{t}^{k} \xi_{t}^{i, j, k}\right)-\theta_{T}^{\prime i}\right)^{+}\right] \\
& =\mathbb{E}\left[\theta_{T}^{i} A_{T}+\sum_{t=0}^{T-1}\left(-\Xi_{t}^{i}+\sum_{(j, k) \in M(i)} \xi_{t}^{i, j, k} Y_{t}^{k}\right) \mathbb{E}\left(A_{T} \mid \mathcal{F}_{t}\right)\right. \\
& \left.+\pi\left(\Delta^{i}+\Pi^{i}\left(\xi^{i}\right)-\sum_{t=0}^{T-1}\left(X_{t}^{i}+\Xi_{t}^{i}\right)\right)^{+}\right] \\
& =\mathbb{E}\left[\theta_{T}^{i} A_{T}+\sum_{t=0}^{T-1}\left(\sum_{(j, k) \in M(i)} \xi_{t}^{i, j, k} Y_{t}^{k}\right) A_{t}+\pi\left(\Gamma^{i}+\Pi^{i}\left(\xi^{i}\right)-\theta_{T}^{i}\right)^{+}\right] \\
& \left.-\mathbb{E}\left(\sum_{t=0}^{T-1} \Xi_{t}^{i} A_{t}\right)\right)
\end{aligned}
$$

which proves the desired result.

We can now turn to the proof of Proposition 7.1.

Proof. Let $\left(A^{*}, S^{*}\right)$ be equilibrium price processes of a standard scheme with strategies $\left(\xi^{*}, \theta^{*}\right)$. Let $\theta^{\prime *}$ be the adjusted optimal strategy as in Lemma 7.2. The assertion follows by checking that conditions (i) to (iii) of Definition 1 are fulfilled by the pair of price processes $\left(A^{*}, S^{\dagger}\right)$ and strategies $\left(\xi^{*}, \theta^{\prime *}\right)$. Since $\theta^{*}$ satisfies the market clearing condition (3.5), so does $\theta^{\prime *}$. This proves (i) while condition (ii) follows directly from (7.3). Moreover, given $\left(\theta^{\prime}, \xi^{i}\right) \in \mathcal{V}^{i}\left(A^{*}\right) \times \mathcal{U}^{i}$, we define strategies $\theta^{i} \in \mathcal{V}^{i}\left(A^{*}\right)$ such that (7.5) and (7.6) hold. According to Proposition 3.1 $A^{*}$ is an integrable martingale and the result of Lemma 7.2 yields

$$
\begin{aligned}
\mathbb{E}\left[H^{A^{*}, S^{\dagger}, i}\left(\theta^{\prime i}, \xi^{i}\right)\right] & =\mathbb{E}\left[L^{A^{*}, S^{*}, i}\left(\theta^{i}, \xi^{i}\right)\right]-\mathbb{E}\left[\sum_{t=0}^{T-1}\left(V_{t}^{i}-\Xi_{t}^{i} A_{t}^{*}\right)\right] \\
& \leq \mathbb{E}\left[L^{A^{*}, S^{*}, i}\left(\theta^{* i}, \xi^{* i}\right)\right]-\mathbb{E}\left[\sum_{t=0}^{T-1}\left(V_{t}^{i}-\Xi_{t}^{i} A_{t}^{*}\right)\right] \\
& =\mathbb{E}\left[H^{A^{*}, S^{\dagger}, i}\left(\theta^{\prime * i}, \xi^{* i}\right)\right]
\end{aligned}
$$


where we used the optimality of the equilibrium strategies $\left(\xi^{*}, \theta^{*}\right)$ of the standard scheme in (7.8). This holds for all $\left(\theta^{\prime}, \xi^{i}\right) \in \mathcal{V}^{i}\left(A^{*}\right) \times \mathcal{U}^{i}$ which proves condition (iii). The converse can be proved in exactly the same way.

Note that not only do allocation prices coincide, but also equilibrium production strategies: $\xi^{\dagger}=\xi^{*}$. Thus the switching costs of the generalized cap-and-trade schemes are the same as for the standard cap-and-trade schemes with adjusted uncontrolled emissions $\left(\Gamma^{i}\right)_{i \in I}$.

7.2. Design of Financial Incentives. In this paragraph we discuss the design of financial incentives that adjust the financial positions of each firm $i \in I$ by

$$
-\sum_{t=0}^{T-1}\left(V_{t}^{i}+\sum_{(j, k) \in M_{i}} \xi_{t}^{i, j, k} Z_{t}^{k}\right)
$$

depending on his production strategy $\xi^{i}$. Obviously, the results of Proposition 7.1 hold in this case. Allowance prices, production strategies, and penalty are identical in equilibrium, to those of the standard scheme. Electricity price is increased by the quantity $Z_{t}^{k}$ at $t=0, \ldots, T-1$. Hence, as depicted in Figure 7.1 the scheme induces a money transfer

$$
\sum_{t=0}^{T-1}\left(V_{t}^{i}+\sum_{(j, k) \in M(i)} \xi_{t}^{i, j, k} Z_{t}^{k}\right)
$$

from producers to the regulator. In the meantime, the quantity

$$
\sum_{t=0}^{T-1} \sum_{(j, k) \in M(i)} \xi_{t}^{i, j, k} Z_{t}^{k}
$$

is entirely passed on to the end consumer, so that $\left(Z_{t}^{k}\right)_{t=0}^{T-1} \geq 0$ results in a money transfer from consumers to the regulator.

REMARK 7. This explains why it is not trivial to reduce windfall profits (and this precise discussion of incentives is needed). At first sight one could e.g. think that windfall profits could be reduced by keeping a book on any traded MWh, marginal technology and carbon price with the objective to charge the producers the windfall profits and reimburse consumers later. The amount that would be charged from each producer $i \in I$ would then depend on his strategy $\xi^{i}$ and the markets product price process $S$ would be given by

$$
\sum_{t=0}^{T-1} \sum_{(j, k) \in M_{i}}\left(S_{t}^{k}-\hat{S}_{t}^{k}\right) \xi_{t}^{i, j, k} .
$$

Obviously under such a regulation the demand will never be satisfied, and hence there exists no equilibrium. On the other side if we change the amount to be charged to

$$
\sum_{t=0}^{T-1} \sum_{(j, k) \in M_{i}}\left(\bar{S}_{t}^{k}-\hat{S}_{t}^{k}\right) \xi_{t}^{i, j, k} .
$$

it follows from above discussion that the entire amount will be passed on to the endconsumer. Thus the windfall profits will not be reduced. 


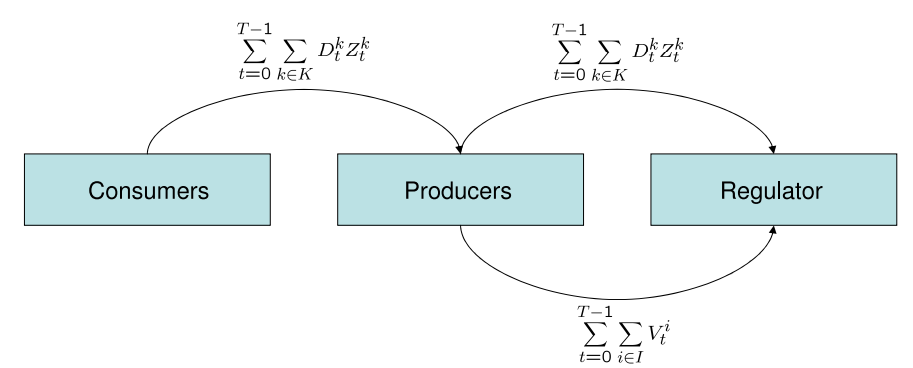

FIGURE 7.1. $V>0$ gives a money transfer from producers to the regulator, while $Z>0$ gives a money transfer from consumer to the regulator. By choosing $V$ and $Z$ in an appropriate way it is possible to avoid a money transfer to/from the regulator.

Proposition 7.1 may look esoteric at first. However, it happens to be very versatile a tool when it comes to designing new schemes with required properties. As corollaries to this proposition, two appropriate adjustments with zero windfall profits are given in Sections 7.3 and 7.4.

7.3. Zero Windfall Profit Scheme with Tax and Subsidy. In this section we consider the generalized allocation scheme given by

$$
\begin{aligned}
X_{t}^{i} & =\Lambda_{0}^{i} 1_{\{t=0\}} \\
Y_{t}^{k} & =0
\end{aligned}
$$

for all $i \in I, k \in K$ and $t=0, \ldots, T-1$. We show here how the tax/subsidy system comprised into the generalized scheme can theoretically lead to zero windfall profits at equilibrium. This result is a direct corollary of Proposition 7.1.

COROLlary 7.3. Consider a generalized cap-and-trade scheme such that for all $i \in I$ and $k \in K$

$$
\begin{aligned}
V_{t}^{i} & =\sum_{(j, k) \in M(i)} \bar{\xi}_{t}^{i, j, k}\left(\bar{S}_{t}-\hat{S}_{t}^{k}\right) \text { for all } t=0, \ldots, T-1 \\
Z_{t}^{k} & =0 \text { for all } k \in K, t=0, \ldots, T-1 .
\end{aligned}
$$

Then each equilibrium $\left(A^{*}, S^{*}\right)$ of the standard cap and trade scheme is also an equilibrium of this generalized scheme. In particular the equilibrium with lowest product prices is given by $(\bar{A}, \bar{S})$ from Theorem 4.6. For this scheme the windfall profits for the aggregated producing sector are zero.

REMARK 8. The processes $\bar{S}, \hat{S}$ and $\bar{\xi}$ that occur in (7.12) are given by Theorem 4.6 and hence independent of the actually realized equilibrium $\left(A^{\dagger}, S^{\dagger}\right)$ and their strategies $\left(\theta^{\dagger}, \xi^{\dagger}\right)$. Hence their computation involves necessarily solving the global optimal control problem. However generalizing Proposition 7.1 easier ways could be found to compute the amount to charge.

To adjust end-consumer costs to a reasonable level, the amount $\sum_{i \in I} \sum_{t=0}^{T-1} V_{t}^{i}$ has to be redistributed from the regulator to the end consumers in an appropriate way. As can be seen in Figure 7.1 this can be omitted for the financial incentives fulfilling

$$
\sum_{t=0}^{T-1} \sum_{i \in I} V_{t}^{i}=-\sum_{t=0}^{T-1} \sum_{k \in K} D_{t}^{k} Z_{t}^{k}
$$


Here there is no money transfer from producers to regulators. Such a setting is discussed in the following Corrolary.

CoROllary 7.4. Consider a generalized cap-and-trade scheme such that for all $i \in I$ and $k \in K$

$$
\begin{aligned}
& V_{t}^{i}=\sum_{(j, k) \in M_{i}} \bar{\xi}_{t}^{i, j, k}\left(\bar{S}_{t}^{k}-\hat{S}_{t}^{k}\right) \quad \text { for all } t=0, \ldots, T-1 \\
& Z_{t}^{k}=-\left(\bar{S}_{t}^{k}-\hat{S}_{t}^{k}\right) \quad \text { for all } t=0, \ldots, T-1
\end{aligned}
$$

where $\bar{S}$ denotes the equilibrium electricity price in the standard scheme, recall Theorem 4.6, and $\hat{S}$ denotes the pure merit order price as defined in (5.3). In this setting each equilibrium $\left(A^{*}, S^{*}\right)$ of the standard cap-and-trade scheme corresponds to an equilibrium of the generalized cap-and-trade scheme given by $\left(A^{*}, S^{*}-(\bar{S}-\hat{S})\right)$. In particular the equilibrium with lowest product price is given by $(\bar{A}, \hat{S})$. In this equilibrium, each producer realizes null windfall profits.

If a firm $i$ follows strategy $\xi^{i}$ its net money transfer to the regulator is given by

$$
\sum_{(j, k) \in M_{i}}\left(\bar{\xi}_{t}^{i, j, k}-\xi_{t}^{i, j, k}\right)\left(\bar{S}_{t}^{k}-\hat{S}_{t}^{k}\right)
$$

Notice that this is zero if a firm follows strategy $\bar{\xi}$. If it decides to produce more or less than in equilibrium, it is rewarded or respectively penalized by (7.14). This increases the incentives to produce and hence results in lower product prices. As shown by (7.13), the only money transfer in this scheme is the transfer of penalty payments from penalized firms to rewarded firms.

7.4. Zero Windfall Profit Scheme with Dynamic Allocation. Here we set $V \equiv Z \equiv 0$, and show how dynamic stochastic allocation of allowances can also lead to null windfall profits for the producers.

Corollary 7.5. Consider a cap and trade scheme with dynamic allowance allocation where

$$
\begin{aligned}
X_{t}^{i} & =-\sum_{(j, k) \in M(i)} \bar{\xi}_{t}^{i, j, k} \frac{\bar{S}_{t}^{k}-\hat{S}_{t}^{k}}{\bar{A}_{t}}+\frac{\Lambda_{0}}{T} \\
Y_{t}^{k} & =\frac{\bar{S}_{t}^{k}-\hat{S}_{t}^{k}}{\bar{A}_{t}}
\end{aligned}
$$

at each time point $t=0, \ldots, T-1$. Here, $\bar{A}$ and $\bar{S}$ denote the equilibrium allowance and lowest electricity price respectively in the standard scheme from Theorem 4.6. Further let $\hat{S}$ be the pure merit order price defined in (5.3). In this setting it holds that $\left(X_{t}^{i}\right)_{t=0}^{T-1} \in \mathcal{L}_{T-1}^{\infty}$ and $\left(Y_{t}^{k}\right)_{t=0}^{T-1} \in \mathcal{L}_{T-1}^{\infty}$ for all $i \in I$ and $k \in K$. Each equilibrium $\left(A^{*}, S^{*}\right)$ of the standard cap-and-trade scheme corresponds to an equilibrium of the generalized cap-and-trade scheme given by $\left(A^{*}, S^{*}-(\bar{S}-\hat{S})\right)$. In particular the equilibrium with lowest prices for goods is given by $\left(A^{*}, \hat{S}\right)$. In this equilibrium, the windfall profits of each firm are zero.

Proof. The equilibrium result is a direct consequence of Proposition 7.1. Hence it remains only to prove that $\left(X_{t}^{i}\right)_{t=0}^{T-1} \in \mathcal{L}_{T-1}^{\infty}$ and $\left(Y_{t}^{i}\right)_{t=0}^{T-1} \in \mathcal{L}_{T-1}^{\infty}$ for all $i \in I$. Let 
us first prove that $\frac{\bar{S}_{t}^{k}-\hat{S}_{t}^{k}}{\bar{A}_{t}}$ is bounded from above. Since

$$
\begin{aligned}
\bar{S}_{t}^{k} & =\max _{i \in I, j \in J^{i, k}}\left(C_{t}^{i, j, k}+e^{i, j, k} \bar{A}_{t}\right) 1_{\left\{\xi_{t}^{i, j, k}>0\right\}} \\
& \leq \max _{i \in I, j \in J^{i, k}} C_{t}^{i, j, k} 1_{\left\{\xi_{t}^{i, j, k}>0\right\}}+\max _{i \in I, j \in J^{i, k}} e^{i, j, k} \bar{A}_{t} 1_{\left\{\xi_{t}^{i, j, k}>0\right\}} \\
& \leq \hat{S}_{t}^{k}+\bar{A}_{t} \max _{i \in I, j \in J^{i, k}} e^{i, j, k}
\end{aligned}
$$

it follows that

$$
\frac{\bar{S}_{t}^{k}-\hat{S}_{t}^{k}}{\bar{A}_{t}} \leq \max _{i \in I, j \in J^{i, k}} e^{i, j, k} \text { for all } k \in K, t=0, \ldots, T-1
$$

which gives the upper bound. To prove the lower bound, we notice that for all $k \in K$ and $t=0, \ldots, T-1$ it holds that $\bar{S}_{t}^{k}-\hat{S}_{t}^{k} \geq 0$ and $\bar{A}_{t} \geq 0$. Thus

$$
\frac{\bar{S}_{t}^{k}-\hat{S}_{t}^{k}}{\bar{A}_{t}} \geq 0 \text { for all } k \in K, t=0, \ldots, T-1
$$

which concludes the proof.

This scheme ensures zero windfall profits at exactly the same reduction costs as for the standard cap and trade scheme, without any extra money transfer from producer to regulator, as e.g. would be the case for auctioning. A further advantage compared to auctioning is that it allows to distribute the amount $\sum_{i \in I} X_{t}^{i}$ differently to control the incentives to build cleaner plants.

The drawback of this scheme is that it requires a random allocation (following a prespecified rule) of allowances dynamically through time. Notice that the daily allocation can be negative for some producers some of the days, this not a problem as can be seen in Green Certificate Schemes.

Notice further that it suffices to give a relative allocation only to those plants that are usually marginal. Therefore in electricity markets the relative allocation does not need to be given to nuclear plants.

The theoretical results of this section suppose market rules that may be uneasy to enforce in a real market. However they show how the different levers brought by the generalized schemes can be used to keep the prices of goods at a low level.

8. Conclusions. In this paper we introduced a new mathematical framework for competitive equilibrium, in which emissions trading schemes can be analyzed. This framework is general enough to accommodate tax based abatement policies, existing cap-and-trade schemes such as those implemented in the first phase of the European Union ETS, as well as new market designs.

The main thrust of the paper is to provide policy makers and regulators with the tools necessary to design and implement cap-and-trade schemes capable of reaching reasonable pollution targets at low social costs while controlling windfall profits and incentives for cleaner production technologies.

On one hand, we develop a rigorous mathematical theory for competitive economic models in which stochastic demand and production costs are given exogenously. We prove existence and uniqueness, of an equilibrium in which price processes for goods and pollution appear endogenously in equilibrium.

On the other hand, we provide analytic and computational tools to analyze and compare the various emissions trading schemes. Regulators and policy makers need 
to understand the structure and the role of these new markets vis-a-vis pollution control, and we view these tools as crucial in the design and the implementation of sound environmental economic policy.

The computational tools that we developed provide, for each market design

- Monte Carlo scenarios generators for equilibrium prices of goods and pollution allowance certificates

- Computation for each scenario of

- pollution levels

- end-consumers costs

- producers windfall profits

- social costs

Finally, as illustration of the versatility of the tools developed for the purpose of the qualitative analysis of cap-and-trade schemes considered in the paper, we implemented them in a case study of the Texas electricity market.

As observed in the SOx and NOx California RECLAIM program and at the end of the first implementation phase of the EU ETS, cap-and-trade systems can fail as too generous an allocation of pollution permits will serve as a disincentive for emissions reductions and will deflate pollution prices. Numerical experiments prove that capand-trade schemes can work in the sense that emissions targets properly chosen can be reached at low costs.

Moreover we use our computational tools to provide a thorough comparison of a sample of alternative schemes: the standard scheme inspired by the first EU implementation phase, an emission tax scheme, and a relative scheme in which allowances distributions are driven by instantaneous (as opposed to historical) production levels. Thereby show that the new allowance allocation scheme which we propose can reduce average windfall profits to zero while keeping reduction cost nearly at the same level as the standards cap and trade scheme. Further this relative scheme allows to control the incentives to build new plants.

The following table summarizes the results of our comparative analysis of standard cap-and-trade schemes (whether or not they including auctioning of allowances) with tax schemes and the relative scheme which we identified in this paper.

\begin{tabular}{|c|c|c|c|c|c|}
\hline \multicolumn{7}{|c|}{ Comparison of Schemes } \\
\hline & Red. Target & Incentives & Windfall & Social Cost & Cons. Cost \\
\hline Standard & + & + & - & + & - \\
\hline Std\&Auct & + & - & - & + & - \\
\hline Relative & + & + & + & + & + \\
\hline Tax & - & - & + & - & - \\
\hline
\end{tabular}

9. Appendix. This final section contains the technical details of the implementations used to produce the numerical results presented in the paper.

9.1. Model Calibration. We chose to run the numerical experiments with data coming from the Texan electricity market, because it forms an independent grid, with few interconnection with other grids. We can thus address the question of the impact of introducing an emission reduction policy in this state without taking into account emission leakage. In subsections 9.1.1 and 9.1.2 electricity demand and fuel switch price process are specified using continuous-time pendants

$$
(D(t))_{t \in[0, T]}, \quad(F(t))_{t \in[0, T]}
$$


for both processes. Note that we write the time parameter in parenthesis instead using subscript, to indicate continuous-time processes. Moreover, the horizon for continuous time is $[0, T]$, where we suppose that the time unit equals to one year. By sampling (9.1) at discrete times, we obtain discrete-time versions of the processes on a daily time step resolution, which is used for numerical computations.

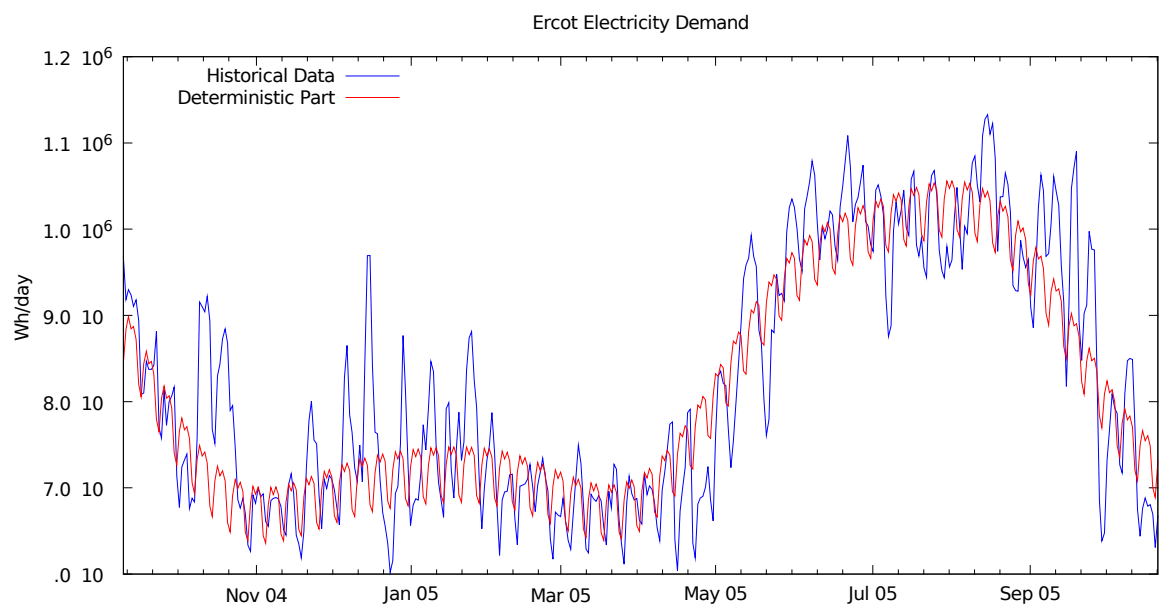

Figure 9.1. Depicted are the historical daily electricity demand for the Ercot supply area from 18/9/04 to 17/9/05 and the corresponding deterministic part $(P(t))_{t=[0, T]}$.

9.1.1. Electricity Demand Process. The continuous-time demand process is modeled by

$$
D(t)=\min \left\{\left(P_{D}(t)+X_{D}(t)\right)^{+}, \kappa^{n}+\kappa^{c}+\kappa^{g}\right\} \quad t \in[0, T]
$$

where the deterministic part

$$
P_{D}(t)=a_{D}+b_{D} t+\sum_{j=0}^{6} c_{j} \cos \left(2 \pi \varphi_{j} t+l_{j}\right) \quad t \in[0, T]
$$

accounts for a linear demand increase superimposed by seasonal and weekly demand fluctuations. The stochastic part $(X(t))_{t \in[0, T]}$ is modeled by an Ornstein-Uhlenbeck process whose evolution follows the stochastic differential equation

$$
d X_{D}(t)=\gamma_{D}\left(\alpha_{D}-X_{D}(t)\right) d t+\sigma_{D} d W(t)
$$

driven by Brownian motion $(W(t))_{t \in[0, T]}$ with parameters $\gamma_{D}, \alpha_{D}, \sigma_{D} \in \mathbb{R}$. The parameter estimation is based on historical load data for the time period $12 / 23 / 03$ 12/23/06 available on ERCOT's website, depicted in the Figure 9.1.1, the parameters of the process were identified in two steps. First the deterministic harmonics (9.2) in the demand process are identified with parameters obtained from peaks in the Fourier transform. Secondly after removing the deterministic part $\left(P_{D}(t)\right)_{t \in[0, T]}$ (red line in this figure) the residual component $\left(X_{D}(t)\right)_{t \in[0, T]}$ is estimated by the standard linear regression described in Subsection 9.1.3. The resulting parameters are:

\begin{tabular}{|c|c|c|}
\hline \multicolumn{3}{|c|}{ Stochastic Part $\left(X_{D}(t)\right)_{t \in[0, T]}$} \\
\hline$\gamma_{D}$ & $\alpha_{D}$ & $\sigma_{D}$ \\
\hline 102 & 0 & 819340 \\
\hline
\end{tabular}




\begin{tabular}{|c|c|c|c|c|c|c|c|c|c|}
\hline \multicolumn{10}{|c|}{ Deterministic Part $\left(P_{D}(t)\right)_{t \in[0, T}$} \\
\hline$a_{D}$ & $b_{D}$ & & $i=0$ & $i=1$ & $i=2$ & $i=3$ & $i=4$ & $i=5$ & $i=6$ \\
\hline 766381 & 38027 & $c_{i}$ & 157526 & 71355 & 19047 & 15056 & 30608 & 16601 & 6364 \\
\hline & & $\varphi_{i}$ & 1 & 2 & 3 & 4 & 52.14 & 104.29 & 208.57 \\
\hline & & $l_{i}$ & -3.56 & -0.82 & -0.83 & -4.36 & 0.77 & -1.55 & -2.15 \\
\hline
\end{tabular}

Notice from $\left(\varphi_{i}\right)_{i=0} 6$ that long term periodicities where computed with a yearly periodic Fourier transform while short term periodicities were computed with a weekly periodic fourier transform.

9.1.2. Fuel Switch Price Process. The continuous-time fuel switch price process is modeled by

$$
F(t)=a_{F}+X_{F}(t) \quad t \in[0, T]
$$

where the stochastic part $\left(X_{F}(t)\right)_{t \in[0, T]}$ is again modeled by an Ornstein-Uhlenbeck process whose evolution follows the stochastic differential equation

$$
d X_{F}(t)=\gamma_{F}\left(\alpha_{F}-X_{F}(t)\right) d t+\sigma_{F} d W(t)
$$

driven by Brownian motion $(W(t))_{t \in[0, T]}$ with parameters $\gamma_{F}, \alpha_{F}, \sigma_{F} \in \mathbb{R}$.

For estimation of the parameters of the fuel spread we used Katy gas spot prices (ICE Katy Exxon Plant Tailgate East Texas) and Platts coal prices (PRB 8400B .35S Dly 1-Mo) from January 2004 to February 2007. Taking into account expected long time gas and coal price we fixed $a=60 \$$ neglecting the recent fuel switch price increase. As for the electricity demand process the parameters of the stochastic component $\left(X_{F}(t)\right)_{t \in[0, T]}$ were calibrated using the procedure described in Subsection 9.1.3. The parameters of the Fuel Switch Price Process are:

\begin{tabular}{|c|c|c|c|}
\hline \multicolumn{4}{|c|}{ Fuel Switch Price Process $(F(t))_{t \in[0, T]}$} \\
\hline$a_{F}$ & $\gamma_{F}$ & $\alpha_{F}$ & $\sigma_{F}$ \\
\hline 60 & 15.26 & 0 & 77.29 \\
\hline
\end{tabular}

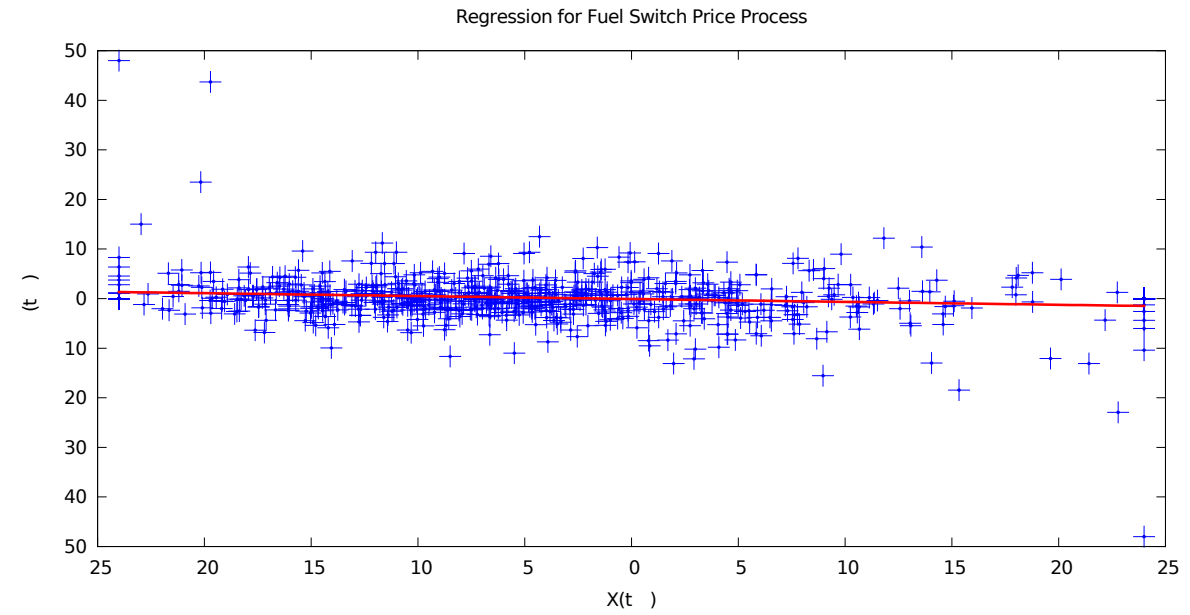

Figure 9.2. Scatter plot of $(X(t \Delta), Y(t \Delta))$ calculated by (9.5) based once historical fuel switch prices for the ercot region. The straight lines depicts the respective estimated linear regressions. 
9.1.3. Linear Regression. The parameters $\gamma_{i}, \alpha_{i}, \sigma_{i}$ for $i \in\{D, F\}$ of the Ornstein-Uhlenbeck processes (9.3) and (9.4) are estimated by a standard linear regression method applied as follows: From the formulas for conditional mean and variance

$$
\begin{aligned}
\mathrm{E}\left(X(t) \mid \mathcal{F}_{s}\right)=X(s) e^{-\gamma(t-s)}+\alpha\left(1-e^{-\gamma(t-s)}\right) & s \leq t \\
\operatorname{Var}\left(X(t) \mid \mathcal{F}_{s}\right)=\frac{\sigma 2}{2 \gamma}\left(1-e^{-2 \gamma(t-s)}\right) & s \leq t
\end{aligned}
$$

we obtain the regression

$$
Y(t \Delta):=X((t+1) \Delta)-X(t \Delta)=\beta_{0}+\beta_{1} X(t \Delta)+\beta_{2} \epsilon_{t} \quad t=1, \ldots, n-1
$$

where $\left(\epsilon_{t}\right)_{t=1}^{n-1}$ are independent, standard Gaussian random variables and $\beta_{0}, \beta_{1}, \beta_{2}$ are connected to $\alpha, \gamma, \sigma$ by

$$
\begin{aligned}
\alpha & =-\frac{\beta_{0}}{\beta_{1}} \\
\gamma & =-\frac{1}{\Delta} \ln \left(1+\beta_{1}\right) \\
\sigma & =\sqrt{\frac{2 \gamma \beta_{2}}{1-e^{-2 \gamma \Delta}}} .
\end{aligned}
$$

\section{REFERENCES}

[1] B. Bueler. Solving an equilibrium model for trade of $\mathrm{CO}_{2}$ emission permits. European Journal of Operational Research, 102(2):393-403, 1997.

[2] M. Cronshaw and J. B. Kruse. Regulated firms in pollution permit markets with banking. Journal of Regulatory Economics, 9(2):179-89, 1996.

[3] J. H. Dales. Pollution, Property and Prices. University of Toronto Press, Toronto, 1968.

[4] M. Fehr and J. Hinz. A quantitative approach to carbon price risk modeling. Preprint, 2006.

[5] H. L. Feng and J. Zhao. Alternative intertemporal permit trading regimes with stochastic abatement costs. Resource and Energy Economics, 28(1):24-40, 2006.

[6] P. L. Joskow, R. B. Schmalensee, and E. Bailey. The market for sulfur dioxide emissions. The American Economic Review, 88(4):669-685, 1998.

[7] P. Leiby and J. Rubin. Intertemporal permit trading for the control of greenhouse gas emissions. Environmental and Resource Economics, 19(3):229-256, 2001.

[8] J. Lemming. Financial risks for green electricity investors and producers in a tradable green certificate market. Energy Policy, 31(1):21-32, 2003.

[9] W. D. Montgomery. Markets in licenses and efficient pollution control programs. Journal of Economic Theory, 5(3):395-418, 1972.

[10] A.P. Robertson and W.S. Robertson. Topological vector spaces. Cambridge Univ. Press, 1973.

[11] J. Rubin. A model of intertemporal emission trading, banking and borrowing. Journal of Environmental Economics and Management, 31(3):269-286, 1996.

[12] S. M. Schennach. The economics of pollution permit banking in the context of title iv of the 1990 clean air act amendments. Journal of Environmental Economics and Management, 40(3):189-21, 2000.

[13] J. Seifert, M. Uhrig-Homburg, and M. Wagner. Dynamic behavior of carbon spot prices .theory and empirical evidence. Preprint, 2006.

[14] J. Sijm, K. Neuhoff, and Y. Chen. Co2 cost pass-through and windfall profits in the power. Climate Policy, 6:4972, 2006.

[15] T. Tietenberg. Environmental Natural and Resource Economics. Addison Wesley, Boston, 2003.

[16] F. Wirl. Consequences of irreversibilities on optimal intertemporal $\mathrm{CO}_{2}$ emission policies under uncertainty. Resource and Energy Economics, 28(2):105-123, 2006. 